\title{
Vector-boson pair production at the $\mathrm{LHC}$ to $\mathcal{O}\left(\alpha^{3}\right)$ accuracy
}

\author{
Anastasiya Bierweiler, Tobias Kasprzik, Johann H. Kühn \\ Karlsruhe Institute of Technology (KIT), Institut für Theoretische Teilchenphysik, \\ D-76128 Karlsruhe, Germany \\ E-mail: kasprzik@particle.uni-karlsruhe.de
}

\begin{abstract}
Building on earlier work on electroweak corrections to W-pair production, the first calculation of the full electroweak one-loop corrections to on-shell $\mathrm{ZZ}, \mathrm{W}^{ \pm} \mathrm{Z}$ and $\gamma \gamma$ production at hadron colliders is presented, explicitly taking into account the full vectorboson mass dependence. As a consequence, our results are valid in the whole energy range probed by LHC experiments. Until now, the electroweak corrections have only been known in dedicated high-energy approximations limited to a specific kinematic regime, in particular requiring high boson transverse momenta. Therefore, our results comprise an important and so far missing ingredient to improve on the theory predictions for these fundamental Standard-Model benchmark processes also at intermediate energies and small scattering angles, where actually the bulk of events is located. In case of Z-pair production we have also included the leptonic decays and the associated weak corrections in our analysis. For this particular channel, corrections of about $-4 \%$ are observed even close to the production threshold. For hard scattering processes with momentum transfers of several hundred GeV one finds large negative corrections which may amount to several tens of percent and lead to significant distortions of transverse-momentum and rapidity distributions.
\end{abstract}




\section{Contents}

1 Introduction 1

2 Details of the calculation 3

2.1 Contributions at leading order 3

2.2 Electroweak radiative corrections 3

2.3 Radiation of massive gauge bosons 4

3 Numerical results $\quad 5$

3.1 Input and setup 5

$\begin{array}{llr}3.2 & \text { Leading-order cross sections } & 8\end{array}$

$\begin{array}{lll}3.3 & \text { Electroweak corrections } & 12\end{array}$

$\begin{array}{lll}\text { 3.3.1 } & \text { Integrated cross sections } & 13\end{array}$

$\begin{array}{lll}\text { 3.3.2 Partially integrated cross sections } & 13\end{array}$

$\begin{array}{lll}\text { 3.3.3 } & \text { Differential distributions } & 15\end{array}$

$\begin{array}{ll}\text { 3.3.4 Comparison with existing results } & 20\end{array}$

3.4 Z-pair production: polarization and decays 22

$\begin{array}{lll}3.4 .1 & \text { Polarization effects } & 22\end{array}$

$\begin{array}{lll}3.4 .2 & \text { Leptonic decays } & 23\end{array}$

3.5 Real-radiation contributions 24

4 Conclusions $\quad 26$

\section{Introduction}

A profound understanding of vector-boson pair production processes at the LHC is desirable for various reasons. Such processes not only contribute an important irreducible background to Standard-Model (SM) Higgs production at moderate energies, but will also provide deeper insight into the physics of the weak interaction at the high-energy frontier, possibly even allowing for the discovery of BSM physics. Consequently, great effort has been made during the last years to push the theory predictions for this process class to a new level, where, besides the dominating QCD corrections, also electroweak (EW) effects have been studied extensively (see, e.g., ref. [1] and references therein).

Extending our work on EW effects in W-pair production at the LHC , we present ${ }^{1}$ corresponding results for on-shell $\mathrm{W}^{ \pm} \mathrm{Z}, \mathrm{ZZ}$ and $\gamma \gamma$ pair production in the $\mathrm{SM}$. We will restrict ourselves to pair production through quark-antiquark annihilation. Photon-photon collisions do not contribute to $\mathrm{WZ}$ production (in contrast to the case of $\mathrm{W}$ pairs), are of higher order for Z-pair and $\gamma$-pair production and will not be discussed further. Also gluon fusion

\footnotetext{
${ }^{1}$ Preliminary results of this investigation have been presented in ref. [2].
} 
is, evidently, irrelevant for $\mathrm{WZ}$ production. For the case of $\mathrm{W}$-pair production we have demonstrated that gluon fusion amounts to order of $5 \%$ relative to the quark-antiquark annihilation with decreasing importance for increasing transverse momenta. Gluon fusion, furthermore, does not lead to a strong modification of the angular and rapidity distributions of the $\mathrm{W}$ bosons. A qualitatively similar behaviour is expected for $\mathrm{ZZ}$ and $\gamma \gamma$ production through gluon fusion, which therefore will not be discussed further. Also QCD corrections for $\mathrm{WZ}$ and $\mathrm{ZZ} / \gamma \gamma$ production are expected to be similar to those for $\mathrm{W}$ pairs discussed in ref. [1] and will not be analyzed in the present paper, which, instead, will be entirely devoted to genuine EW corrections.

Earlier papers have emphasized the drastic influence of the EW corrections on cross sections and distributions in the high- $p_{\mathrm{T}}$ region. However, only pair-production channels with at least one massive gauge boson were considered [3-5] while a phenomenological analysis of the $\gamma \gamma$ channel is still missing. To close this gap, the first computation of the full EW corrections to photon-pair hadroproduction is presented in this work. Moreover, the above papers neglect terms of order $M_{V}^{2} / \hat{s}$. Also this issue will be addressed in the present paper. All the processes under consideration are affected by large corrections, amounting to several tens, sometimes up to fifty percent. (This fact has even triggered studies of next-to-next-to-leading logarithmic corrections for W-pair production at the LHC [6], which, however, where not extended to Z-pair or WZ production.) Nevertheless, significant differences are observed between the different final states, as far as the size of the corrections is concerned. We, furthermore, investigate rapidity distributions, both for small and for large invariant masses of the diboson system and observe significant distortions that could be misinterpreted as anomalous triple-boson couplings. The full mass dependence, namely terms proportional to powers of $M_{V}^{2} / \hat{s}$, is consistently accounted for to obtain results valid in the whole energy range probed by LHC experiments, in particular for $V$ pairs produced near threshold ${ }^{2}$ or at low transverse momenta. Therefore, our results are complementary to those presented in refs. [3, 4], where only logarithmic corrections were considered, but the leptonic decays of the vector bosons and related off-shell effects were included in a double-pole approximation. Comparing both approaches, we try to estimate the remaining theoretical uncertainties related to EW corrections in this important process class.

To investigate this aspect in more detail, also the combined production and decay process for $\mathrm{Z}$ pairs is calculated, including NLO corrections. To simplify the discussion, only the four-lepton state $\mathrm{e}^{+} \mathrm{e}^{-} \mu^{+} \mu^{-}$is considered. Excellent agreement with the results of Accomando et al. is observed in the region where the high-energy approximation employed in ref. [4] is valid. We, furthermore, investigate the contributions from the different helicity configurations including NLO corrections and observe that the transverse polarization not only dominates completely at large $p_{\mathrm{T}}$, it is also the most prominent configuration as far as the total cross section is concerned.

In addition, we also study the impact of real gauge-boson radiation leading to three-

\footnotetext{
${ }^{2}$ Although EW corrections are expected to be small at low energies, for Z-pair production a shift of about $-4 \%$ is observed even close to threshold, whereas the corresponding corrections are below 1 percent in the remaining cases.
} 
boson final states. In principle, these configurations might compensate some of the negative contributions from virtual corrections, in practice, however, real radiation is significantly smaller than virtual contributions.

\section{Details of the calculation}

\subsection{Contributions at leading order}

At the $\mathrm{LHC}, \mathrm{WW}, \mathrm{W}^{ \pm} \mathrm{Z}, \mathrm{ZZ}$ and $\gamma \gamma$ production is, at lowest order $\mathcal{O}\left(\alpha^{2}\right)$, induced by the partonic processes

$$
\begin{aligned}
q \bar{q} & \rightarrow \mathrm{W}^{-} \mathrm{W}^{+} \quad(q=\mathrm{u}, \mathrm{d}, \mathrm{s}, \mathrm{c}, \mathrm{b}), \\
\mathrm{u}_{i} \overline{\mathrm{d}}_{j} & \rightarrow \mathrm{W}^{+} \mathrm{Z}, \quad \overline{\mathrm{u}}_{i} \mathrm{~d}_{j} \rightarrow \mathrm{W}^{-} \mathrm{Z} \quad(i=1,2 ; j=1,2,3), \\
q \bar{q} & \rightarrow \mathrm{ZZ} \\
q \bar{q} & \rightarrow \gamma \gamma .
\end{aligned}
$$

where the corresponding LO partonic cross sections are evaluated according to

$$
\hat{\sigma}_{\mathrm{LO}}^{q \bar{q}^{\prime} \rightarrow V_{1} V_{2}}=\frac{1}{2 \hat{s} N_{q \bar{q}^{\prime}}} \int \mathrm{d} \Phi\left(V_{1} V_{2}\right) \sum_{\text {col spin pol }} \sum_{\text {s. }}\left|\mathcal{M}_{0}^{q \bar{q}^{\prime} \rightarrow V_{1} V_{2}}\right|^{2},
$$

with the tree-level helicity amplitudes $\mathcal{M}_{0}^{q \bar{q}^{\prime} \rightarrow V_{1} V_{2}}$, the two-particle phase-space measure $\mathrm{d} \Phi\left(V_{1} V_{2}\right)$ and the averaging factor $N_{q \bar{q}^{\prime}}=36$. It is understood that for ZZ and $\gamma \gamma$ production the cross sections receive an additional symmetry factor of $1 / 2$. Note that one finds $\int \mathrm{d} \xi_{V}\left(\mathrm{~d} \sigma^{\mathrm{pp} \rightarrow V V} / \mathrm{d} \xi_{V}\right)=2 \sigma^{\mathrm{pp} \rightarrow V V}$ for $\xi=p_{\mathrm{T}}$ or $\xi=y$ for these particular channels.

\subsection{Electroweak radiative corrections}

To allow for consistent predictions with full $\mathcal{O}\left(\alpha^{3}\right)$ accuracy, virtual EW corrections as well as real corrections due to photon radiation have to be considered. The evaluation of the radiative corrections is based on the well-established FeynArts/FormCalc/LoopTools setup [7-11], and all processes have been independently cross-checked by a setup based on QGraf [12] and Form [13].

To considerably reduce the computational effort, light quark masses are neglected whenever possible. However, soft and collinear singularities occurring in intermediate steps of the calculation are regularized by small quark masses $m_{q}$ and an infinitesimal photon mass $\lambda$, generating unphysical $\ln m_{q}$ and $\ln \lambda$ terms. To allow for a numerically stable evaluation of those infrared(IR)-divergent parts of the cross sections related to real radiation, the phase-space slicing method is adopted as detailed in ref. [14] for mass regularization. Finally, adding real and virtual contributions, the regulator-mass dependence drops out in any properly defined physical result. However, in complete analogy to QCD, residual collinear singularities attributed to initial-state (IS) radiation survive and have to be absorbed in renormalized PDFs in a proper factorization procedure adding the collinear counterterm defined by eq. (3.16) of ref. [15]. In the present computation, we apply the $\overline{\mathrm{MS}}$ factorization scheme for the QED factorization. 
The input parameters to be specified in Section 3.1 are renormalized in a modified on-shell scheme [16], where the Fermi constant $G_{\mu}$ is used instead of $\alpha(0)$ to effectively account for universal corrections induced by the running of $\alpha(\mu)$ to the weak scale [14]. However, for the computation of the $\gamma \gamma$ channel, $\alpha(0)$ is used as input for the EW coupling, since the corresponding radiative corrections do not receive universal contributions related to the running of the coupling constant. According to the previous considerations, the partonic cross section at $\mathcal{O}\left(\alpha^{3}\right)$ accuracy may be written as

$$
\hat{\sigma}_{\mathrm{NLO}}^{q \bar{q}^{\prime} \rightarrow V_{1} V_{2}(\gamma)}=\hat{\sigma}_{\mathrm{LO}}^{q \bar{q}^{\prime} \rightarrow V_{1} V_{2}}+\hat{\sigma}_{\mathrm{LO}}^{q \bar{q}^{\prime} \rightarrow V_{1} V_{2} \gamma}+\hat{\sigma}_{\text {virt }}^{q \bar{q}^{\prime} \rightarrow V_{1} V_{2}}
$$

where the different NLO contributions are given by

$$
\begin{aligned}
\hat{\sigma}_{\mathrm{LO}}^{q \bar{q}^{\prime} \rightarrow V_{1} V_{2} \gamma} & =\frac{1}{2 \hat{s} N_{q \bar{q}^{\prime}}} \int \mathrm{d} \Phi\left(V_{1} V_{2} \gamma\right) \sum_{\text {col }} \sum_{\text {spin pol }} \sum_{\text {pol }}\left|\mathcal{M}_{0}^{q \bar{q}^{\prime} \rightarrow V_{1} V_{2} \gamma}\right|^{2} \\
\hat{\sigma}_{\text {virt }}^{q \bar{q}^{\prime} \rightarrow V_{1} V_{2}} & =\frac{1}{2 \hat{s} N_{q \bar{q}^{\prime}}} \int \mathrm{d} \Phi\left(V_{1} V_{2}\right) \sum_{\text {col spin pol }} \sum_{1} \sum_{2} \operatorname{Re}\left\{\left(\mathcal{M}_{0}^{q \bar{q}^{\prime} \rightarrow V_{1} V_{2}}\right)^{*} \mathcal{M}_{1}^{q \bar{q}^{\prime} \rightarrow V_{1} V_{2}}\right\}
\end{aligned}
$$

with the properly renormalized one-loop amplitudes $\mathcal{M}_{1}^{q \bar{q}^{\prime} \rightarrow V_{1} V_{2}}$. The hadronic results at the LHC are then obtained by convoluting the partonic cross sections with appropriately chosen PDFs and summing incoherently over all contributing channels,

$$
\sigma_{\mathrm{NLO}}^{\mathrm{pp} \rightarrow V_{1} V_{2}(\gamma)}=\int_{\tau_{0}}^{1} \mathrm{~d} \tau \int_{\tau}^{1} \frac{\mathrm{d} x_{b}}{x_{b}} \sum_{q, \bar{q}^{\prime}} f_{q / \mathrm{p}}\left(x_{a}, \mu_{\mathrm{F}}^{2}\right) f_{\bar{q}^{\prime} / \mathrm{p}}\left(x_{b}, \mu_{\mathrm{F}}^{2}\right) \hat{\sigma}_{\mathrm{NLO}}^{q \bar{q}^{\prime} \rightarrow V_{1} V_{2}(\gamma)}\left(\tau s, \mu_{\mathrm{F}}^{2}\right),
$$

where the hadronic CM energy $s$ is related to $\hat{s}$ via $\hat{s}=\tau s$, with $\tau=x_{a} x_{b}$. The kinematic production threshold of a vector-boson pair in the final state is reflected by the choice of the lower integration boundary $\tau_{0}=\left(M_{V_{1}}+M_{V_{2}}\right)^{2} / s$, corresponding to a minimal partonic CM energy of $\hat{s}_{0}=\tau_{0} s$. The factorization scale $\mu_{\mathrm{F}}$ enters the partonic cross section through the redefinition of the PDFs in the QED factorization procedure described above.

\subsection{Radiation of massive gauge bosons}

As in ref. [1] we study the effect of massive boson radiation on the massive vector-boson pair production cross section, where the additional massive boson is treated fully exclusively in the event selection to allow for a robust estimate of the corresponding phenomenological effects. This is motivated by the possibility that the logarithmically enhanced positive contributions from the radiation of an additional soft or collinear massive gauge boson might compensate the negative virtual corrections, as has been argued recently for $\mathrm{Z}+$ jet production [17].

To be specific, we take into account the following partonic channels, where the corresponding partonic cross sections have to be computed according to

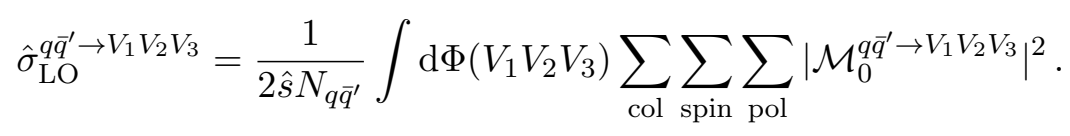


In case of ZZ pair production, the contributions from the processes

$$
\begin{aligned}
q \bar{q} & \rightarrow \mathrm{ZZZ}, \\
q \bar{q}^{\prime} & \rightarrow \mathrm{ZZW}^{ \pm}
\end{aligned}
$$

have to be considered, where the two Zs with highest $p_{\mathrm{T}}$ have to fulfill the LO cuts to be specified in section 3.1, while the third massive particle is treated inclusively. To assess the real-radiation effects in $\mathrm{W}^{ \pm} \mathrm{Z}$ production, we compute the cross sections for

$$
\begin{aligned}
q \bar{q} & \rightarrow \mathrm{W}^{ \pm} Z \mathrm{~W}^{\mp}, \\
q \bar{q}^{\prime} & \rightarrow \mathrm{W}^{ \pm} \mathrm{ZZ} .
\end{aligned}
$$

In the first case the $\mathrm{W}^{\mp}$ is treated inclusively, in the second case the $\mathrm{Z}$ with lowest $p_{\mathrm{T}}$. In section 3.5, numerical results will be presented for massive boson radiation normalized to the LO $q \bar{q}$-induced pair-production channels,

$$
\delta_{V_{1} V_{2} V_{3}}=\sigma_{\mathrm{LO}}^{\mathrm{pp} \rightarrow V_{1} V_{2} V_{3}} / \sigma_{\mathrm{LO}}^{\mathrm{pp} \rightarrow V_{1} V_{2}}-1,
$$

as well as for hard photon radiation (i.e. $p_{\mathrm{T}, \gamma}>15 \mathrm{GeV},\left|y_{\gamma}\right|<2.5$ ),

$$
\delta_{V_{1} V_{2} \gamma}=\sigma_{\mathrm{LO}}^{\mathrm{pp} \rightarrow V_{1} V_{2} \gamma} / \sigma_{\mathrm{LO}}^{\mathrm{pp} \rightarrow V_{1} V_{2}}-1 .
$$

\section{Numerical results}

In this section, for the first time the full EW corrections to Z- and $\gamma$-pair production as well as $\mathrm{W}^{ \pm} \mathrm{Z}$ production are presented. Most of the discussion is focussed on LHC8 and LHC14. For completeness, we also show cross sections for these processes at the Tevatron with a $\mathrm{CM}$ energy of $\sqrt{s}=1.96 \mathrm{TeV}$. The corresponding results for $\mathrm{W}$-pair production originally presented in ref. [1] are given for comparison. In the following, the relative effects $\delta_{\mathrm{EW}}$ of the EW corrections are defined as

$$
\delta_{\mathrm{EW}}=\frac{\sigma_{\mathrm{NLO}}^{\mathrm{pp} \rightarrow V_{1} V_{2}(\gamma)}}{\sigma_{\mathrm{LO}}^{\mathrm{pp} \rightarrow V_{1} V_{2}}}-1 .
$$

\subsection{Input and setup}

For the computation presented here the same setup as specified in ref. [1] is applied. To be specific, we use the following SM input parameters for the numerical analysis,

$$
\begin{aligned}
& G_{\mu}=1.16637 \times 10^{-5} \mathrm{GeV}^{-2}, \\
& M_{\mathrm{W}}=80.398 \mathrm{GeV}, \quad M_{\mathrm{Z}}=91.1876 \mathrm{GeV} \text {, } \\
& M_{\mathrm{H}}=125 \mathrm{GeV}, \quad M_{\mathrm{t}}=173.4 \mathrm{GeV} \text {. }
\end{aligned}
$$

For the evaluation of all tree-level contributions we assume a block-diagonal CKM matrix with

$$
\left|V_{\mathrm{ud}}\right|=\left|V_{\mathrm{cs}}\right|=0.974, \quad\left|V_{\mathrm{us}}\right|=\left|V_{\mathrm{cd}}\right|=\sqrt{1-\left|V_{\mathrm{ud}}\right|^{2}} .
$$


Ignoring, furthermore, quark masses within the first two families, both tree-level and oneloop predictions for ZZ and $\gamma \gamma$ are equivalent to those without quark mixing. As a consequence of the smallness of the bottom-quark PDF the tree-level contribution from $b \bar{b}$ annihilation to ZZ or $\gamma \gamma$ is small to start with. In addition, the non-diagonal CKM elements involving b quarks are small, and the ansatz (3.3) is well justified. As a consequence, $\mathrm{b} \overline{\mathrm{b}} \rightarrow \mathrm{ZZ}$ or $\gamma \gamma$ can safely be handled within the third family. ${ }^{3}$ The situation is different for the WZ channel. In this case, the interplay between CKM angles and PDFs leads to a shift of the tree-level prediction of about one percent. For the radiative corrections the CKM matrix can, therefore, still be set to unity.

In the on-shell scheme applied in our computation, the weak mixing angle $\cos ^{2} \theta_{\mathrm{w}}=$ $M_{\mathrm{W}}^{2} / M_{\mathrm{Z}}^{2}$ is a derived quantity. For the computation of the processes (2.1) and the corresponding EW radiative corrections, we use the MSTW2008LO PDF set [18] in the LHAPDF setup [19]. In order to consistently include $\mathcal{O}(\alpha)$ corrections, in particular real radiation with the resulting collinear singularities, PDFs in principle should take these QED effects into account. Such a PDF analysis has been performed in ref. [20], and the $\mathcal{O}(\alpha)$ effects are known to be small, as far as their effect on the quark distribution is concerned [21]. In addition, the currently available PDFs incorporating $\mathcal{O}(\alpha)$ corrections [20] include QCD effects at NLO, whereas our EW analysis is $\mathrm{LO}$ with respect to perturbative QCD only. For these reasons, the MSTW2008LO set is used as our default choice for the quark-induced processes. Our default choice for the factorization scale is the average of the vector-boson transverse masses

$$
\mu_{\mathrm{F}}=\overline{m_{\mathrm{T}}}=\frac{1}{2}\left(\sqrt{M_{V_{1}}^{2}+p_{\mathrm{T}, V_{1}}^{2}}+\sqrt{M_{V_{2}}^{2}+p_{\mathrm{T}, V_{2}}^{2}}\right) .
$$

A similar scale choice was taken in ref. [4] for the computation of the EW corrections to four-lepton production at the LHC. Yet we point out that the relative EW corrections, which are the main subject of this paper, only depend on the choice of $\mu_{\mathrm{F}}$ at the subpercent level even for large transverse momenta.

In our default setup, we require a minimum transverse momentum and a maximum rapidity for the final-state vector bosons,

$$
p_{\mathrm{T}, V_{i}}>15 \mathrm{GeV}, \quad\left|y_{V_{i}}\right|<2.5, \quad i=1,2,
$$

to define a $V$-boson pair production event. Thereby we exclude events where the bosons are emitted collinear to the initial-state partons, which for the $\gamma \gamma$ channel would inevitably lead to collinear singularities in the LO cross section. However, for final states with massive gauge bosons selected numerical results will also be presented without applying the above cuts.

For the definition of a two-photon final state we require at least two visible photons fulfilling the acceptance cuts (3.5). If additional photon bremsstrahlung is present, any further phase-space cuts will only be applied to the two visible photons with highest $p_{\mathrm{T}}$, while the third $\gamma$ is treated inclusively to ensure IR safety.

\footnotetext{
${ }^{3}$ We point out that a non-vanishing top-quark mass is consistently included in the computation of the one-loop contributions discussed in this paper.
} 

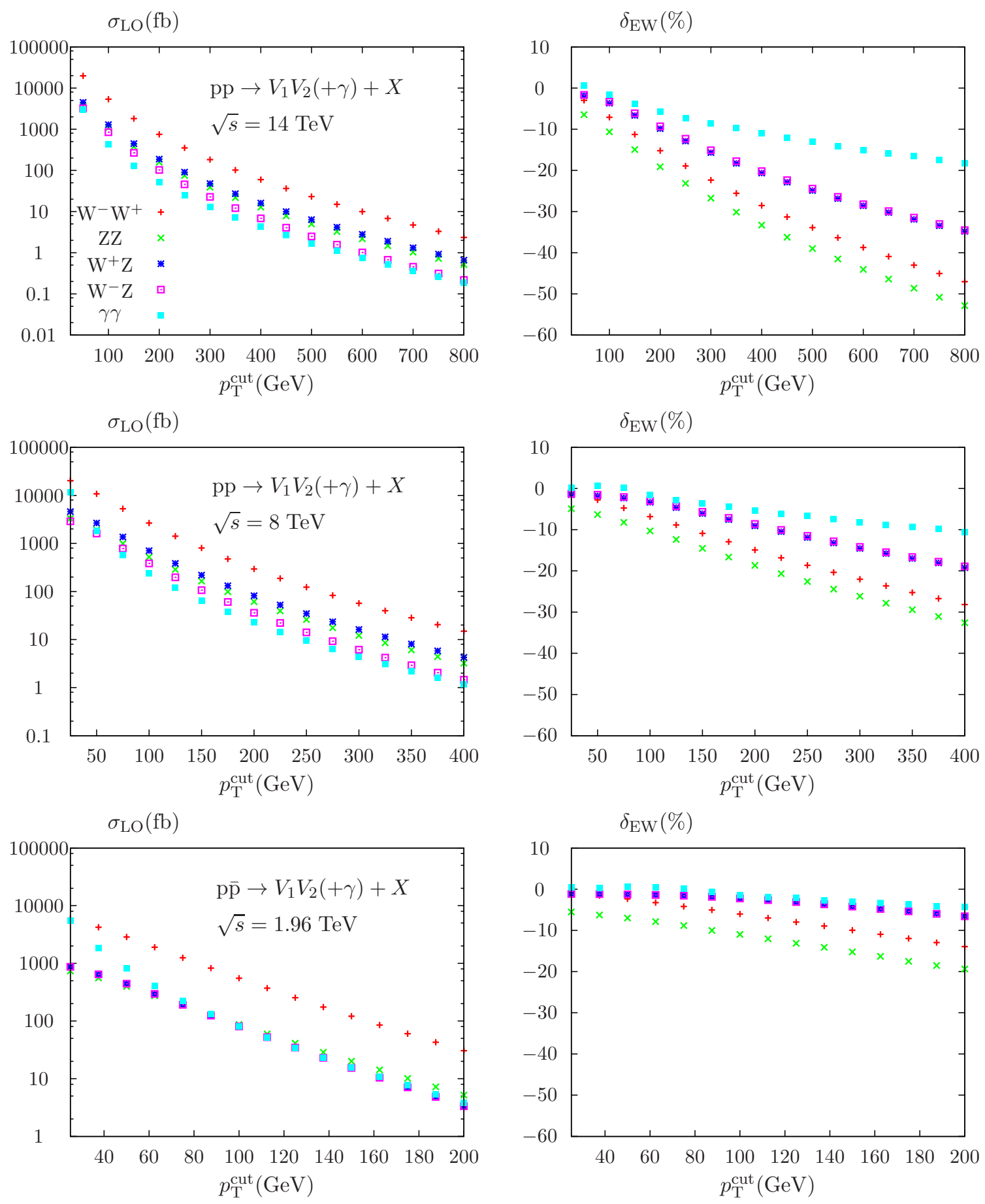

Figure 1. Integrated LO cross sections (left) and relative EW corrections (right) evaluated with our default setup for different cuts on the transverse momenta of the final-state bosons. 

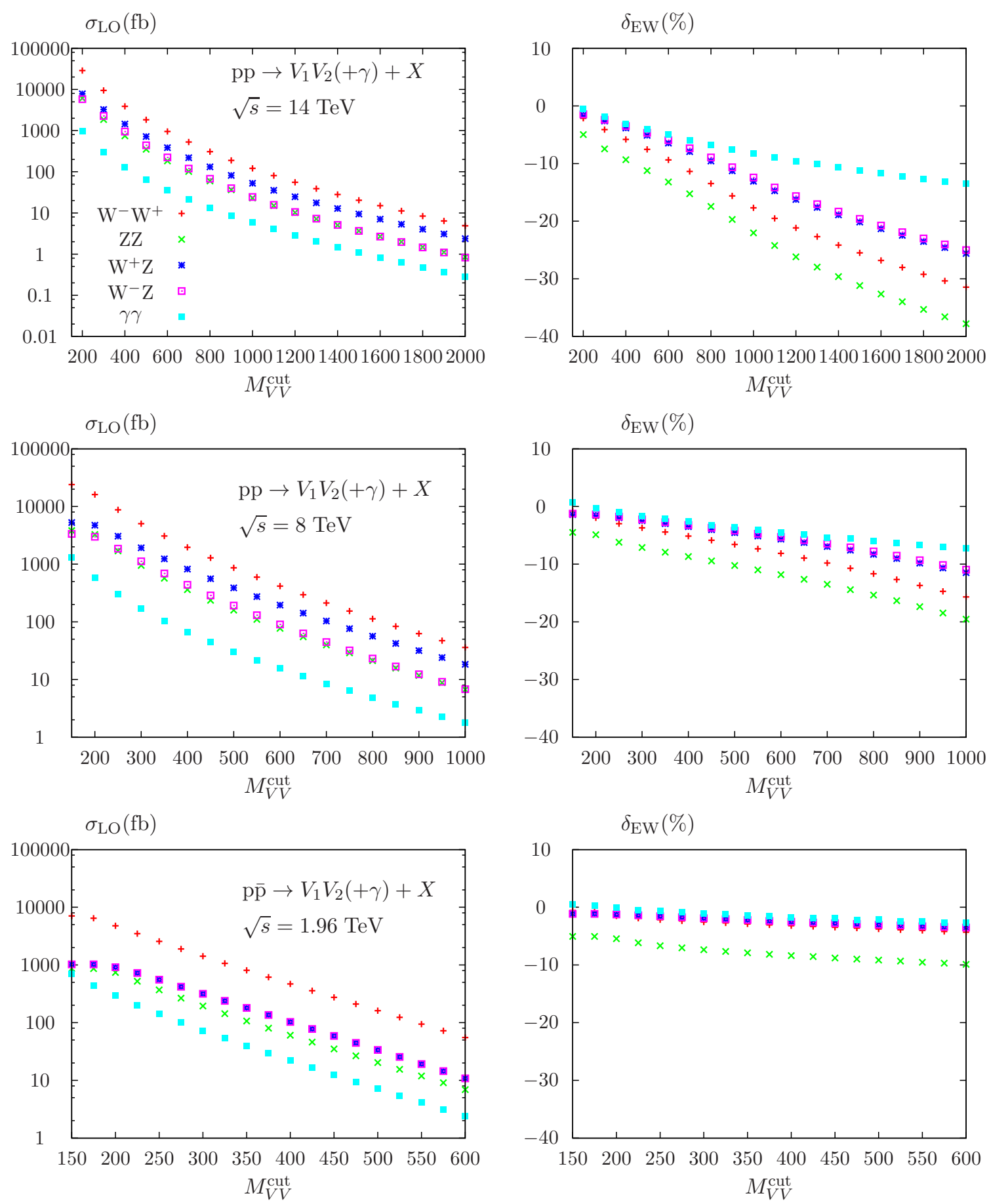

Figure 2. Integrated LO cross sections (left) and relative EW corrections (right) evaluated with our default setup for different cuts on the invariant mass of the final-state bosons. The respective results are presented for LHC14 (top), LHC8 (center) and the Tevatron (bottom).

\subsection{Leading-order cross sections}

Before discussing the EW corrections to $V$-pair production at hadron colliders in detail let us first recall the most striking features at leading order. 


\begin{tabular}{|c||c|c|c|c|c|c|c|c|c|c|}
\hline \multicolumn{1}{|c||}{} & \multicolumn{2}{c|}{$\mathrm{W}^{-} \mathrm{W}^{+}$} & \multicolumn{2}{c|}{$\mathrm{ZZ}$} & \multicolumn{2}{c|}{$\mathrm{W}^{+} \mathrm{Z}$} & \multicolumn{2}{c|}{$\mathrm{W}^{-} \mathrm{Z}$} & \multicolumn{2}{c|}{$\gamma \gamma$} \\
default cuts & $\sigma_{\mathrm{LO}}(\mathrm{pb})$ & $\delta_{\mathrm{EW}}(\%)$ & $\sigma_{\mathrm{LO}}(\mathrm{pb})$ & $\delta_{\mathrm{EW}}(\%)$ & $\sigma_{\mathrm{LO}}(\mathrm{pb})$ & $\delta_{\mathrm{EW}}(\%)$ & $\sigma_{\mathrm{LO}}(\mathrm{pb})$ & $\delta_{\mathrm{EW}}(\%)$ & $\sigma_{\mathrm{LO}}(\mathrm{pb})$ & $\delta_{\mathrm{EW}}(\%)$ \\
\hline \hline LHC8 & 23.99 & -0.7 & 3.810 & -4.4 & 5.256 & -1.4 & 3.343 & -1.2 & 41.38 & 0.2 \\
LHC14 & 42.39 & -0.9 & 7.066 & -4.5 & 8.677 & -1.5 & 6.463 & -1.3 & 62.69 & 0.2 \\
\hline Tevatron & 7.054 & -0.5 & 0.8624 & -4.9 & 1.023 & -1.1 & 1.023 & -1.1 & 19.21 & 0.2 \\
\hline
\end{tabular}

Table 1. Integrated leading-order cross sections and relative EW corrections for the LHC and the Tevatron evaluated with the default setup defined in Section 3.1.

\begin{tabular}{|c||c|c|c|c|c|c|c|c|}
\hline \multicolumn{1}{|c||}{} & \multicolumn{2}{c|}{$\mathrm{W}^{-} \mathrm{W}^{+}$} & \multicolumn{2}{c|}{$\mathrm{ZZ}$} & \multicolumn{2}{c|}{$\mathrm{W}^{+} \mathrm{Z}$} & \multicolumn{2}{c|}{$\mathrm{W}^{-} \mathrm{Z}$} \\
no cuts & $\sigma_{\mathrm{LO}}(\mathrm{pb})$ & $\delta_{\mathrm{EW}}(\%)$ & $\sigma_{\mathrm{LO}}(\mathrm{pb})$ & $\delta_{\mathrm{EW}}(\%)$ & $\sigma_{\mathrm{LO}}(\mathrm{pb})$ & $\delta_{\mathrm{EW}}(\%)$ & $\sigma_{\mathrm{LO}}(\mathrm{pb})$ & $\delta_{\mathrm{EW}}(\%)$ \\
\hline \hline LHC8 & 35.51 & -0.4 & 5.064 & -4.1 & 8.273 & -1.4 & 4.643 & -1.3 \\
LHC14 & 75.02 & -0.4 & 11.02 & -4.2 & 17.11 & -1.4 & 10.65 & -1.3 \\
\hline Tevatron & 7.916 & -0.2 & 0.9466 & -4.7 & 1.123 & -1.1 & 1.123 & -1.1 \\
\hline
\end{tabular}

Table 2. Total leading-order cross sections and relative EW corrections for the LHC and the Tevatron evaluated without any phase-space cuts.

\begin{tabular}{|c||c|c|c|c|c|c|c|c|}
\hline \multicolumn{8}{|c|}{$\mathrm{pp} \rightarrow V_{1} V_{2}(+\gamma)+X$ at $\sqrt{s}=14 \mathrm{TeV}$} \\
\cline { 1 - 8 } $\begin{array}{c}\text { default cuts } \\
p_{\mathrm{T}}^{\text {cut }}(\mathrm{GeV})\end{array}$ & $\sigma_{\mathrm{LO}}(\mathrm{pb})$ & $\delta_{\mathrm{EW}}(\%)$ & $\sigma_{\mathrm{LO}}(\mathrm{pb})$ & $\delta_{\mathrm{EW}}(\%)$ & $\sigma_{\mathrm{LO}}(\mathrm{pb})$ & $\delta_{\mathrm{EW}}(\%)$ & $\sigma_{\mathrm{LO}}(\mathrm{pb})$ & $\delta_{\mathrm{EW}}(\%)$ \\
\hline \hline 50 & 3.660 & -6.3 & 4.498 & -1.9 & 3.228 & -1.7 & 2.979 & 0.6 \\
100 & 1.087 & -10.4 & 1.296 & -3.7 & 0.849 & -3.3 & 0.432 & -1.6 \\
250 & $7.495 \times 10^{-2}$ & -23.0 & $90.56 \times 10^{-2}$ & -12.9 & $4.583 \times 10^{-2}$ & -12.3 & $2.451 \times 10^{-2}$ & -7.0 \\
500 & $49.89 \times 10^{-4}$ & -38.9 & $63.64 \times 10^{-4}$ & -24.9 & $24.79 \times 10^{-4}$ & -24.4 & $16.95 \times 10^{-4}$ & -13.0 \\
750 & $72.16 \times 10^{-5}$ & -50.6 & $92.43 \times 10^{-5}$ & -33.3 & $31.25 \times 10^{-5}$ & -33.0 & $25.66 \times 10^{-5}$ & -17.3 \\
1000 & $14.60 \times 10^{-5}$ & -60.1 & $18.35 \times 10^{-5}$ & -39.8 & $57.32 \times 10^{-6}$ & -39.6 & $54.91 \times 10^{-6}$ & -20.6 \\
1250 & $35.28 \times 10^{-6}$ & -68.4 & $42.92 \times 10^{-6}$ & -45.1 & $12.86 \times 10^{-6}$ & -45.1 & $14.15 \times 10^{-6}$ & -23.5 \\
1500 & $94.73 \times 10^{-7}$ & -75.7 & $10.99 \times 10^{-6}$ & -49.6 & $32.48 \times 10^{-7}$ & -49.6 & $40.71 \times 10^{-7}$ & -25.9 \\
\hline
\end{tabular}

Table 3. Integrated leading-order cross sections and relative EW corrections at LHC14 for different cuts on the minimal boson transverse momenta.

LO results for the processes $\mathrm{pp}(\overline{\mathrm{p}}) \rightarrow V_{1} V_{2}+X$ (see eqs. (2.1)) are given in table 1 for integrated cross sections evaluated with our default setup, in table 2 for the total cross sections without any cuts. The relative EW corrections are also displayed and will be discussed in detail in section 3.3. The rates for $\mathrm{ZZ}$ and $\mathrm{W}^{-} \mathrm{Z}$ production are in the same ballpark, both at the LHC and the Tevatron. For the LHC, being a proton collider, the cross section for $\mathrm{W}^{+} \mathrm{Z}$ production is larger than for $\mathrm{W}^{-} \mathrm{Z}$ production, while at the Tevatron they trivially coincide. The WW production cross sections, however, are roughly a factor of 5 (7) larger at the LHC (Tevatron) than those for ZZ production. For our default cuts, the cross section for $\gamma \gamma$ production is even larger than the one for $\mathrm{W}$-pair production, as a consequence of the singular behaviour for small $p_{\mathrm{T}, \gamma}$.

Requiring tighter cuts on the boson transverse momenta (tables 3 and 5 and figure 1 , 


\begin{tabular}{|c||c|c|c|c|c|c|c|c|}
\hline \multicolumn{8}{|c|}{$\mathrm{pp} \rightarrow V_{1} V_{2}(+\gamma)+X$ at $\sqrt{s}=14 \mathrm{TeV}$} \\
\hline $\begin{array}{c}\text { default cuts } \\
M_{V V}^{\text {cut }}(\mathrm{GeV})\end{array}$ & $\sigma_{\mathrm{LO}}(\mathrm{pb})$ & $\delta_{\mathrm{EW}}(\%)$ & $\sigma_{\mathrm{LO}}(\mathrm{pb})$ & $\delta_{\mathrm{EW}}(\%)$ & $\sigma_{\mathrm{LO}}(\mathrm{pb})$ & $\delta_{\mathrm{EW}}(\%)$ & $\sigma_{\mathrm{LO}}(\mathrm{pb})$ & $\delta_{\mathrm{EW}}(\%)$ \\
\hline \hline 200 & 6.094 & -5.0 & 7.820 & -1.6 & 5.790 & -1.5 & 0.966 & -0.4 \\
300 & 1.859 & -7.4 & 3.252 & -2.6 & 2.273 & -2.4 & 0.304 & -1.8 \\
500 & 0.352 & -11.2 & 0.716 & -5.1 & 0.440 & -4.7 & $64.54 \times 10^{-3}$ & -3.8 \\
1000 & $23.10 \times 10^{-3}$ & -22.0 & $52.70 \times 10^{-3}$ & -13.1 & $24.47 \times 10^{-3}$ & -12.4 & $58.63 \times 10^{-4}$ & -8.1 \\
1500 & $36.65 \times 10^{-4}$ & -31.2 & $94.50 \times 10^{-4}$ & -20.1 & $36.67 \times 10^{-4}$ & -19.6 & $11.09 \times 10^{-4}$ & -11.0 \\
2000 & $84.75 \times 10^{-5}$ & -37.8 & $23.73 \times 10^{-4}$ & -25.6 & $82.87 \times 10^{-5}$ & -25.0 & $28.25 \times 10^{-5}$ & -13.4 \\
2500 & $23.17 \times 10^{-5}$ & -43.4 & $68.36 \times 10^{-5}$ & -30.2 & $22.52 \times 10^{-5}$ & -29.6 & $84.20 \times 10^{-6}$ & -15.4 \\
3000 & $69.60 \times 10^{-6}$ & -48.2 & $16.72 \times 10^{-5}$ & -35.0 & $67.72 \times 10^{-6}$ & -33.5 & $27.49 \times 10^{-6}$ & -17.0 \\
\hline
\end{tabular}

Table 4. Integrated leading-order cross sections and relative EW corrections at LHC14 for different cuts on the minimal boson invariant mass.

\begin{tabular}{|c|c|c|c|c|c|c|c|c|}
\hline \multicolumn{9}{|c|}{$\mathrm{pp} \rightarrow V_{1} V_{2}(+\gamma)+X$ at $\sqrt{s}=8 \mathrm{TeV}$} \\
\hline default cuts & \multicolumn{2}{|l|}{$\mathrm{ZZ}$} & \multicolumn{2}{|c|}{$\mathrm{W}^{+} \mathrm{Z}$} & \multicolumn{2}{|l|}{$\mathrm{W}^{-} \mathrm{Z}$} & \multicolumn{2}{|l|}{$\gamma \gamma$} \\
\hline$p_{\mathrm{T}}^{\text {cut }}(\mathrm{GeV})$ & $\sigma_{\mathrm{LO}}(\mathrm{pb})$ & $\delta_{\mathrm{EW}}(\%)$ & $\sigma_{\mathrm{LO}}(\mathrm{pb})$ & $\delta_{\mathrm{EW}}(\%)$ & $\sigma_{\mathrm{LO}}(\mathrm{pb})$ & $\delta_{\mathrm{EW}}(\%)$ & $\sigma_{\mathrm{LO}}(\mathrm{pb})$ & $\delta_{\mathrm{EW}}(\%)$ \\
\hline 50 & 1.913 & -6.2 & 2.651 & -1.8 & 1.610 & -1.5 & 1.843 & 0.6 \\
\hline 100 & 0.523 & -10.2 & 0.706 & -3.4 & 0.384 & -3.0 & 0.242 & -1.3 \\
\hline 150 & 0.164 & -14.5 & 0.219 & -6.1 & 0.106 & -5.7 & $64.40 \times 10^{-3}$ & -3.5 \\
\hline 250 & $26.08 \times 10^{-3}$ & -22.5 & $34.70 \times 10^{-3}$ & -11.9 & $14.10 \times 10^{-3}$ & -11.5 & $95.15 \times 10^{-4}$ & -6.7 \\
\hline 350 & $60.94 \times 10^{-4}$ & -29.3 & $80.73 \times 10^{-4}$ & -17.0 & $29.08 \times 10^{-4}$ & -16.6 & $22.17 \times 10^{-4}$ & -9.3 \\
\hline 500 & $10.11 \times 10^{-4}$ & -38.1 & $13.11 \times 10^{-4}$ & -23.2 & $42.29 \times 10^{-5}$ & -22.9 & $38.01 \times 10^{-5}$ & -12.3 \\
\hline 600 & $35.40 \times 10^{-5}$ & -43.2 & $44.99 \times 10^{-5}$ & -26.6 & $13.87 \times 10^{-5}$ & -26.5 & $13.77 \times 10^{-5}$ & -14.2 \\
\hline 750 & $83.51 \times 10^{-6}$ & -50.1 & $10.18 \times 10^{-5}$ & -31.1 & $30.31 \times 10^{-6}$ & -31.1 & $34.62 \times 10^{-6}$ & -16.5 \\
\hline
\end{tabular}

Table 5. Integrated leading-order cross sections and relative EW corrections at LHC8 for different cuts on the minimal boson transverse momenta.

\begin{tabular}{|c||c|c|c|c|c|c|c|c|}
\hline \multicolumn{8}{|c|}{$\mathrm{pp} \rightarrow V_{1} V_{2}(+\gamma)+X$ at $\sqrt{s}=8 \mathrm{TeV}$} \\
\cline { 1 - 3 } $\begin{array}{c}\text { default cuts } \\
M_{V V}^{\text {cut }}(\mathrm{GeV})\end{array}$ & $\sigma_{\mathrm{LO}}(\mathrm{pb})$ & $\delta_{\mathrm{EW}}(\%)$ & $\sigma_{\mathrm{LO}}(\mathrm{pb})$ & $\delta_{\mathrm{EW}}(\%)$ & $\sigma_{\mathrm{LO}}(\mathrm{pb})$ & $\delta_{\mathrm{EW}}(\%)$ & $\sigma_{\mathrm{LO}}(\mathrm{pb})$ & $\delta_{\mathrm{EW}}(\%)$ \\
\hline \hline 200 & 3.271 & -4.9 & 4.728 & -1.5 & 2.980 & -1.4 & 0.579 & -0.3 \\
300 & 0.950 & -7.1 & 1.921 & -2.5 & 1.117 & -2.2 & 0.169 & -1.6 \\
400 & 0.360 & -8.7 & 0.821 & -3.5 & 0.439 & -3.2 & $66.45 \times 10^{-3}$ & -2.6 \\
500 & 0.159 & -10.2 & 0.387 & -4.5 & 0.191 & -4.2 & $30.52 \times 10^{-3}$ & -3.6 \\
700 & $39.70 \times 10^{-3}$ & -13.5 & 0.103 & -6.9 & $44.69 \times 10^{-3}$ & -6.5 & $84.71 \times 10^{-4}$ & -5.2 \\
800 & $21.27 \times 10^{-3}$ & -15.4 & $56.47 \times 10^{-3}$ & -8.3 & $23.07 \times 10^{-3}$ & -7.8 & $48.66 \times 10^{-4}$ & -5.8 \\
1000 & $67.00 \times 10^{-4}$ & -19.5 & $18.35 \times 10^{-4}$ & -11.5 & $68.07 \times 10^{-4}$ & -11.0 & $17.91 \times 10^{-4}$ & -7.1 \\
1200 & $23.88 \times 10^{-4}$ & -23.7 & $68.65 \times 10^{-4}$ & -14.4 & $23.82 \times 10^{-4}$ & -14.0 & $72.84 \times 10^{-5}$ & -8.3 \\
1500 & $61.33 \times 10^{-5}$ & -28.5 & $18.45 \times 10^{-4}$ & -18.2 & $60.60 \times 10^{-5}$ & -17.6 & $21.25 \times 10^{-5}$ & -9.8 \\
\hline
\end{tabular}

Table 6. Integrated leading-order cross sections and relative EW corrections at LHC8 for different cuts on the minimal boson invariant mass.

left) and on invariant masses (tables 4 and 6 and figure 2, left), one observes a rapid decrease of the cross sections over several orders of magnitude, corresponding to the fact 

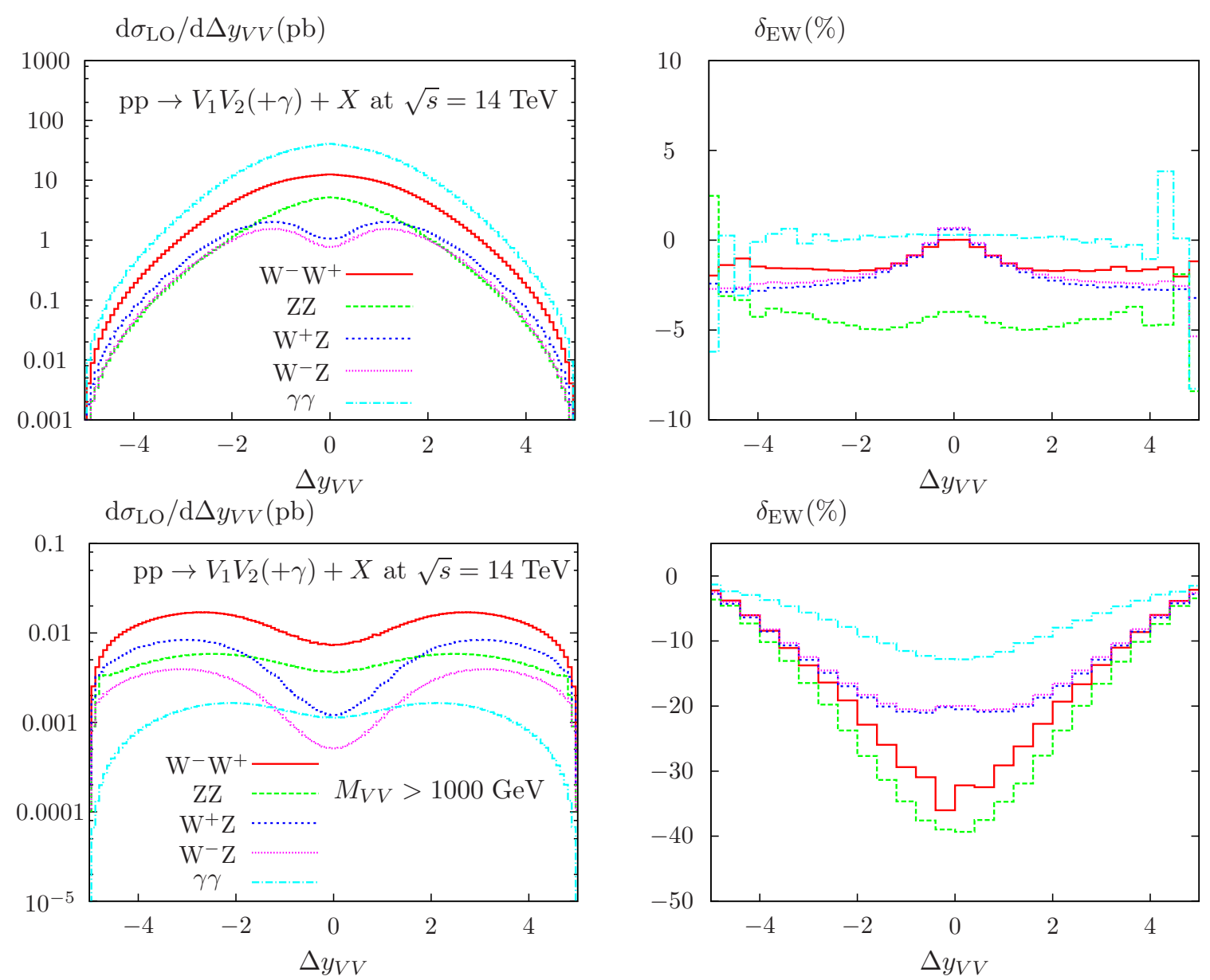

Figure 3. Differential LO distributions of the boson rapidity gap (left) and corresponding EW corrections (right) at LHC14, evaluated with our default setup (top) and with a minimal boson invariant mass of $1000 \mathrm{GeV}$ (bottom).

that $V$-pair production is dominated by both small scattering angles and low-energetic events, as has already been pointed out in ref. [1] for the WW case. Consequently, a cut on the transverse momentum leads to a much stronger reduction than a comparable cut on the invariant mass, $M_{V V}^{\text {cut }}=2 p_{\mathrm{T}}^{\text {cut }}$, as is obvious from tables 3 and 4 . In addition, also the relative rates of the different channels change dramatically. While comparable at low $p_{\mathrm{T}}$, the cross section for $\mathrm{W}^{-} \mathrm{Z}$ production is 3 times smaller than the one for $\mathrm{W}^{+} \mathrm{Z}$ production when going to large values of $p_{\mathrm{T}}$ and $M_{V V}$. For large $p_{\mathrm{T}}$, ZZ production behaves similar to $\mathrm{W}^{+} \mathrm{Z}$ production, at large invariant masses the event rates are comparable to $\mathrm{W}^{-} \mathrm{Z}$ production. In contrast, the $\gamma \gamma$ cross section, which dominates at low transverse momenta, drops rapidly for high invariant masses, at high transverse momenta it is comparable to $\mathrm{W}^{-} \mathrm{Z}$ production.

For high invariant masses the forward-backward peaking of the cross sections is even more pronounced than for low energies as can be deduced from the left plots of figures 3 and 4 , where the differential cross sections are presented as a function of the boson rapidity 

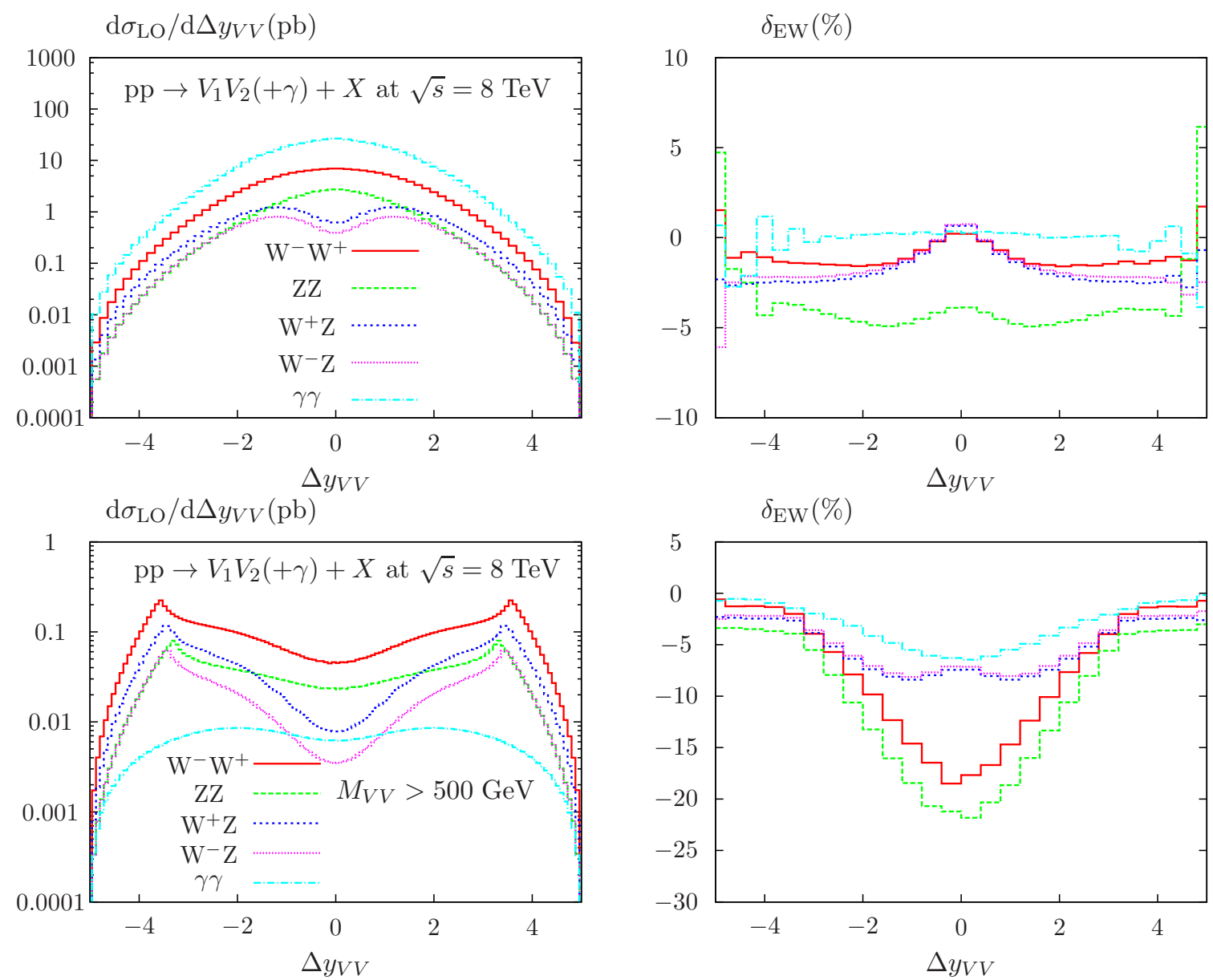

Figure 4. Differential LO distributions of the boson rapidity gap (left) and corresponding EW corrections (right) at LHC8, evaluated with our default setup (top) and with a minimal boson invariant mass of $500 \mathrm{GeV}$ (bottom).

gap $\Delta y_{V V}=y_{V_{1}}-y_{V_{2}}$, evaluated with our default cuts (top) and with a minimal invariant mass of $1000 \mathrm{GeV}(500 \mathrm{GeV})$ at LHC14 (LHC8) (bottom). Note that $\Delta y_{V V}$ for fixed $M_{V V}$ corresponds to the scattering angle in the diboson rest frame. At low energies the distributions reach their maximum in the central region, i.e. at rather low values of $\left|\Delta y_{V V}\right|$, corresponding to central events, at high invariant masses they become maximal at $\left|\Delta y_{V V}\right| \simeq$ 3 , corresponding to a drastic peaking in the forward and backward directions.

\subsection{Electroweak corrections}

This section will be devoted to a detailed numerical analysis of the EW corrections to vector-boson pair production at the LHC and Tevatron. The corresponding effects on the total cross sections will be discussed in section 3.3.1, those for partially integrated cross sections in different kinematic regimes in section 3.3.2. In section 3.3.3, we discuss the EW corrections to differential distributions of observables relevant at the LHC, namely transverse momenta, rapidities and invariant masses. In particular, we focus on the structure 
of the respective corrections at highest energies, where high- as well as low- $p_{\mathrm{T}}$ production of vector-boson pairs is addressed separately.

\subsubsection{Integrated cross sections}

In a first step we present the EW corrections for the total production cross sections for $\mathrm{WW}, \mathrm{W}^{ \pm} \mathrm{Z}, \mathrm{ZZ}$ and $\gamma \gamma$ in table 1, using the default cuts described before. The EW corrections amount to roughly one percent, with the $\mathrm{ZZ}$ channel being the only exception with its sizable negative correction of about $-4 \%$. As discussed below, this feature is present already close to production threshold, applies to Tevatron, LHC8 and LHC14 and is also present in rapidity distributions, as long as we do not enforce large $\hat{s}$ through selected cuts.

For reference purpose we also list the results for the total cross section without any cuts for all final states apart of $\gamma \gamma$, where the corresponding cross sections would diverge (table 2). The size of the radiative corrections remains practically unchanged.

\subsubsection{Partially integrated cross sections}

In order to enhance hard scattering events and explore the $\mathrm{TeV}$ region it is useful to consider partially integrated cross sections, where additional cuts are introduced on the transverse momenta of the gauge bosons or on the invariant mass of the gauge-boson pair. Numerical results are listed in tables 3, 4, 5 and 6 and in figures 1 and 2 .

As expected, one observes increasingly negative corrections with increasing $p_{\mathrm{T}}^{\text {cut }}$ or $M_{V V}^{\text {cut }}$. The corrections are largest for $\mathrm{ZZ}$ production, reaching $-50 \%$ at $\mathrm{LHC} 14$ for $p_{\mathrm{T}}^{\text {cut }}=$ $800 \mathrm{GeV}$, and smallest for $\gamma \gamma$ production, where they remain below $20 \%$ for all kinematic configurations. As already stated in our paper on WW production, the EW corrections at the LHC are considerably more important than at the Tevatron.

For illustration we also present the results up to $p_{\mathrm{T}}^{\text {cut }}=1 \mathrm{TeV}$ which might be accessible in a high-luminosity run. In this case corrections exceeding $40 \%$ are observed in $\mathrm{W}$-pair production and the question of two-loop contributions necessarily arises. For W-pair production the logarithmically enhanced (NNLL) corrections have been discussed in ref. [6], for the other final states they are not yet available and we will not dwell further on this subject.

The particularly large negative EW corrections in case of Z-pair production may be understood as a consequence of two completely independent physical effects. On the one hand, the EW corrections to the $\mathrm{ZZ}$ cross section exhibit a constant offset of $-4 \%$ irrespective of cuts when compared to WW, WZ and $\gamma \gamma$ production. This feature is most obvious in figure 5 , where the $M_{V V}^{\text {cut }}$ dependence of the cross sections is displayed without additional rapidity or transverse-momentum restrictions, i.e. without the default cuts described in section 3.1. Sudakov-enhanced logarithms cannot be made responsible, since a $-4 \%$ correction is already present in the threshold region, and it is hardly dependent on the actual value of the collider energy. We additionally observe that real radiation of hard photons, which is included in our results together with radiation of soft and virtual photons, is particularly small for ZZ production (see figure 12, bottom). Taking both effects into account, the EW corrections at high $p_{\mathrm{T}}$ which can be attributed to large logarithms 

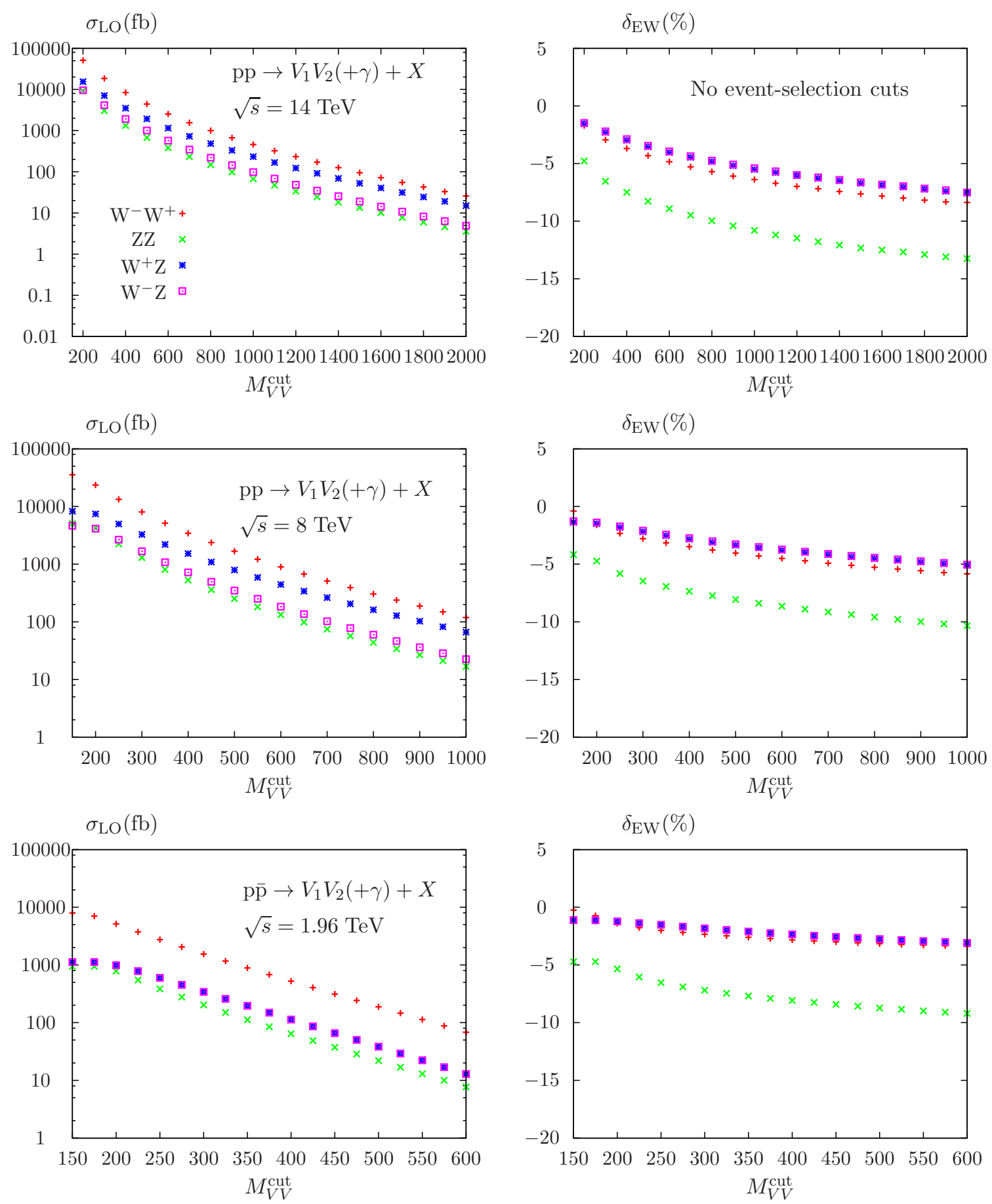

Figure 5. Integrated LO cross sections (left) and relative EW corrections (right) for different cuts on the invariant mass of the boson pair; no additional cuts on rapidity or transverse momentum are applied. The respective results are presented for LHC14 (top), LHC8 (center) and the Tevatron (bottom). 
stemming from the weak corrections are similar for $\mathrm{WW}$ and $\mathrm{ZZ}$ production, while they are significantly smaller in case of $\mathrm{W}^{ \pm} \mathrm{Z}$ production.

Nevertheless, also corrections to $\mathrm{W}^{-} \mathrm{Z}$ production are sizable, reaching $-35 \%$ at LHC14 for $p_{\mathrm{T}}^{\text {cut }}=800 \mathrm{GeV}$. Furthermore they are quite similar to those for $\mathrm{W}^{+} \mathrm{Z}$ production, since at parton level the corresponding unpolarized cross sections coincide. Small deviations solely arise from the different parton-luminosities multiplying the real-radiation and virtual contributions, respectively, leading to slightly different relative corrections, since the differential partonic corrections $\hat{\delta}_{\mathrm{EW}}(\hat{s}, \hat{t})$, which evidently are the same for both channels, enter the hadronic results with different weights.

Going back to figure 5, we find that the relative corrections are small (below 10\%) even for large invariant masses (without additional cuts on rapidities and transverse momenta), since vector-boson pair production is dominated by low scattering angles, i.e. small $|\hat{t}|$, where no logarithmically enhanced EW corrections are expected. Surprisingly enough, even moderate cuts on the boson transverse momenta and rapidities lead to considerable relative corrections as shown in figure 2 .

Another interesting finding is that the EW corrections in case of $\gamma \gamma$ production are again substantially smaller than in the massive channels, reaching $-20 \%$ for $p_{\mathrm{T}}^{\text {cut }}=1000 \mathrm{GeV}$, which is consistent with corresponding results from ref. [22], where logarithmic corrections to the processes $\gamma \gamma \rightarrow f \bar{f}$ were computed in the high- $p_{\mathrm{T}}$ approximation. ${ }^{4}$ As already stated above, up to now no phenomenological study at all exists of the EW effects in photon-pair production at hadron colliders.

\subsubsection{Differential distributions}

In this section differential cross sections for various kinematic scenarios are investigated. Specifically, in addition to transverse-momentum, invariant-mass and rapidity distributions generated in our default setup, we also present results with explicit cuts on the invariant mass of the vector bosons. This allows to investigate the EW corrections in the high-energy regime where new-physics signatures might have a sizable impact. As will be shown, significant distortions of the angular distributions are observed which might easily be misinterpreted as a signal of anomalous couplings and hence new physics.

In figures 6, 7 and 8 we display the differential distributions and corresponding EW corrections for the boson transverse-momentum, invariant-mass and rapidity distributions, respectively, evaluated in the default setup defined in section 3.1. As already stated for the partially integrated cross sections in the previous section, the LO distributions rapidly decrease with increasing values of $p_{\mathrm{T}}$ and $M_{V V}$, reflecting that vector-boson pair production is in general dominated by events with low $\hat{s}$, as a consequence of the rapidly-falling PDFs and cross sections. At the same time, the EW corrections increase with $p_{\mathrm{T}}$ and $M_{V V}$, ranging between $-15 \%$ for $\gamma \gamma$ and $-45 \%$ for $\mathrm{ZZ}$ if we consider $p_{\mathrm{T}}$ values of $800 \mathrm{GeV}$ at LHC14 as example.

The relative corrections for the rapidity distributions (figure 8, right) are small for WW, WZ and $\gamma \gamma$ production and resemble the corrections for the integrated cross sections

\footnotetext{
${ }^{4}$ The full one-loop EW corrections to $\gamma \gamma \rightarrow \mathrm{t} \overline{\mathrm{t}}$ were presented even earlier in ref. [23].
} 

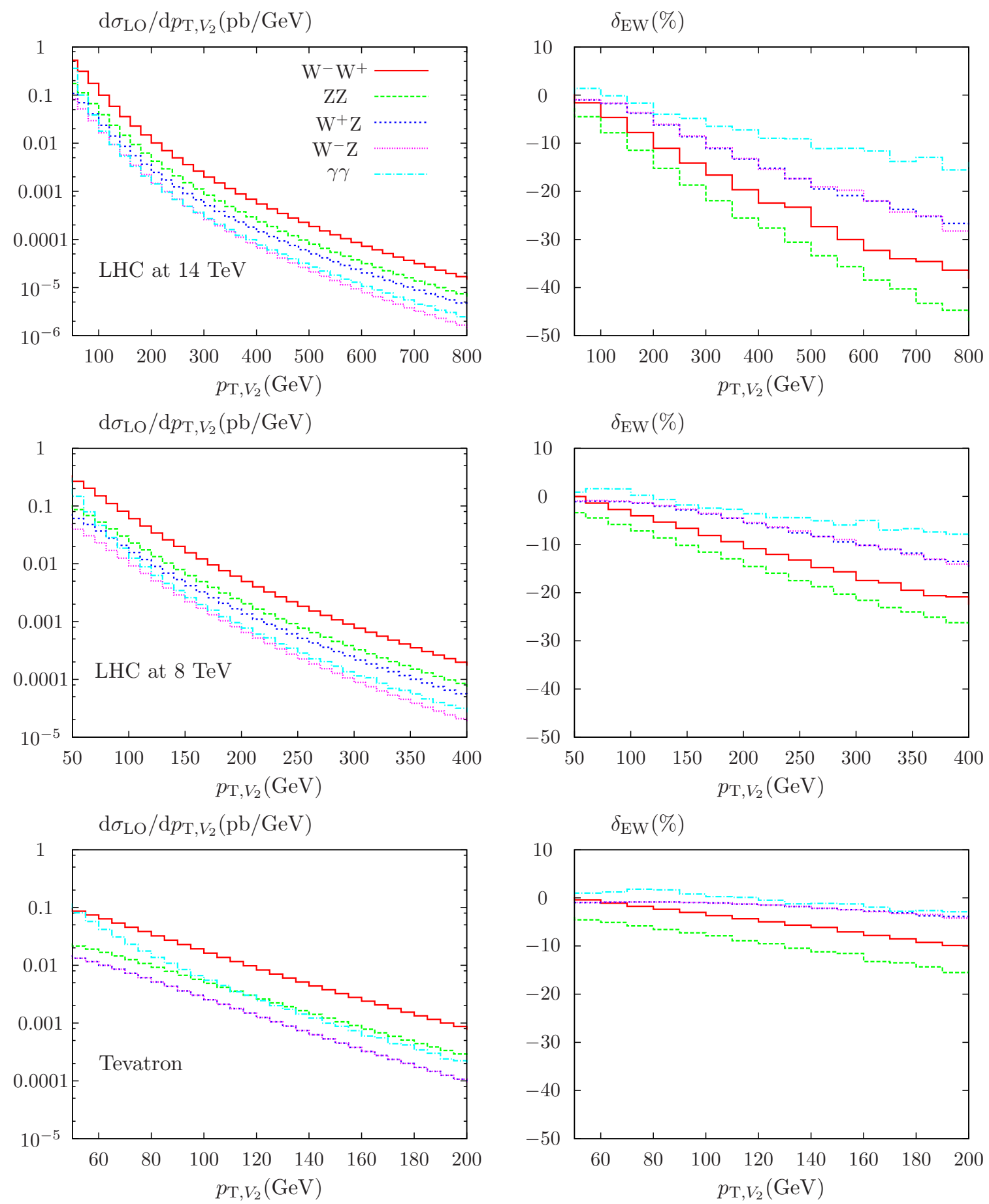

Figure 6. Differential LO distributions of the transverse momentum (left) and corresponding relative EW corrections (right) evaluated with our default setup. The respective results are presented for LHC14 (top), LHC8 (center) and the Tevatron (bottom).

presented in table 1. Again, for $\mathrm{ZZ}$ production a constant offset of $-4 \%$ in the EW corrections is evident, reflecting a remarkably constant $K$-factor. This behaviour is expected, 

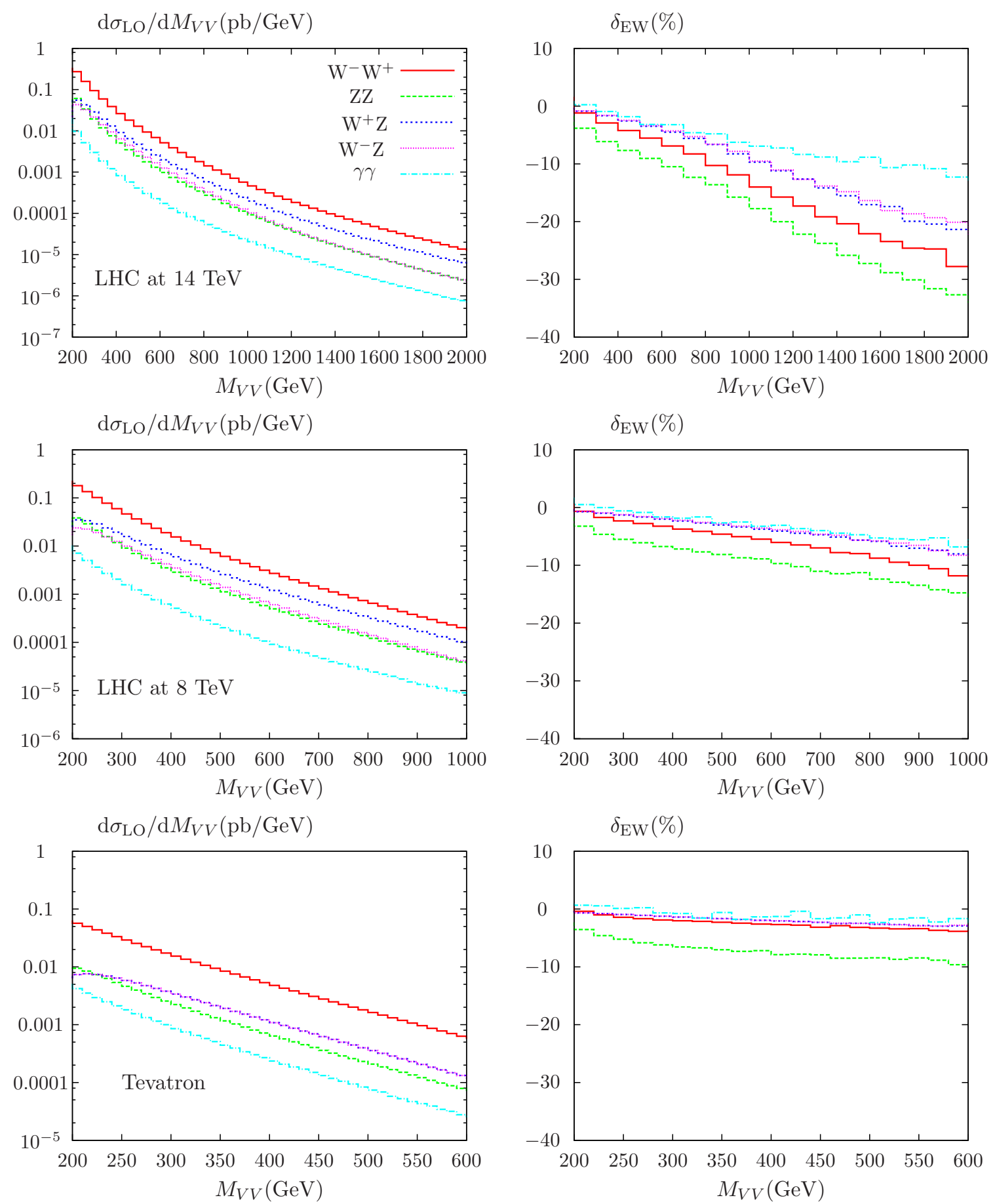

Figure 7. Differential LO distributions of the invariant mass (left) and corresponding relative EW corrections (right) evaluated with our default setup. The respective results are presented for LHC14 (top), LHC8 (center) and the Tevatron (bottom).

since both the total cross section and the rapidity distributions are dominated by small $M_{V V}$. 

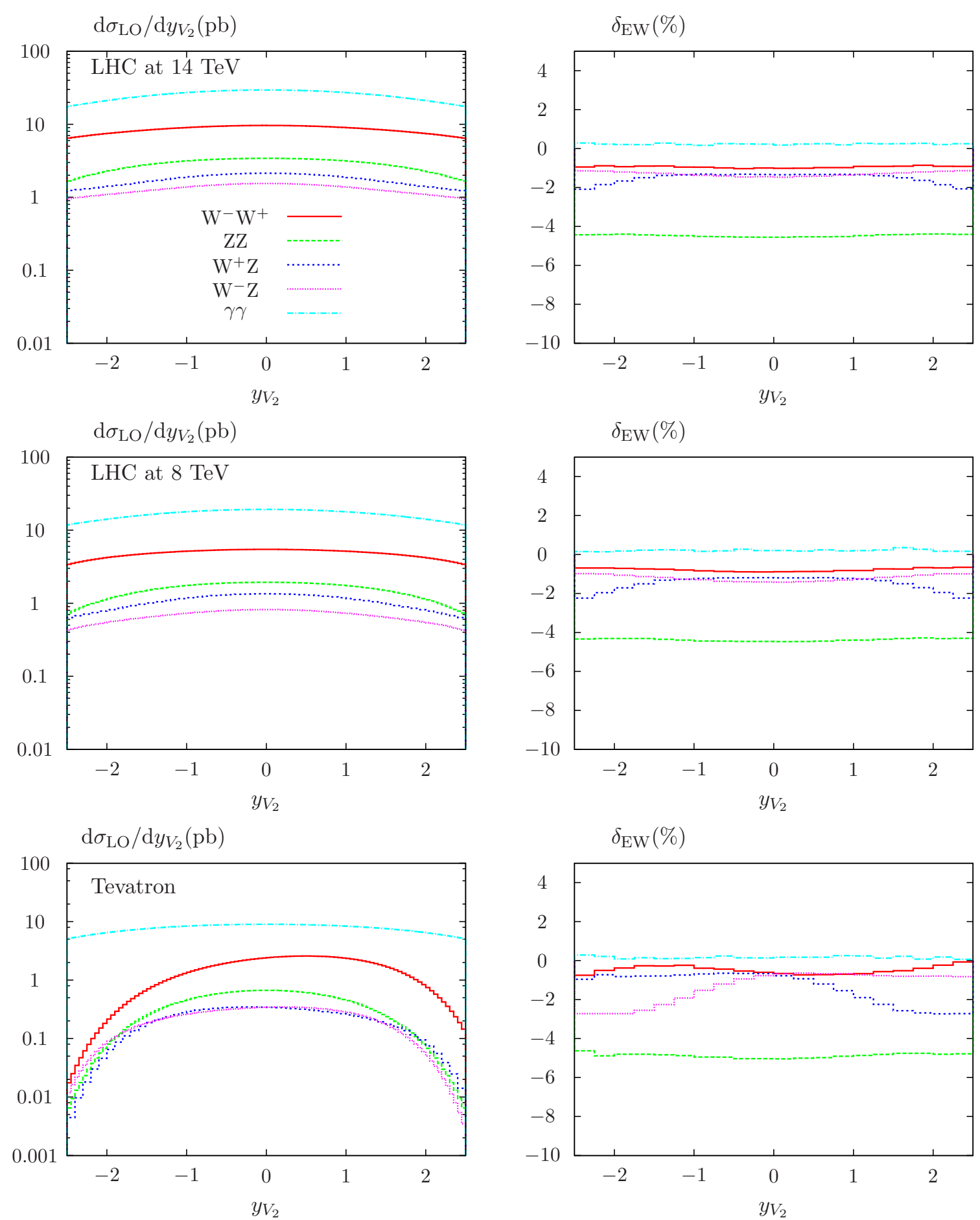

Figure 8. Differential LO distributions of the rapidity (left) and corresponding relative EW corrections (right) evaluated with our default setup. The respective results are presented for LHC14 (top), LHC8 (center) and the Tevatron (bottom). 

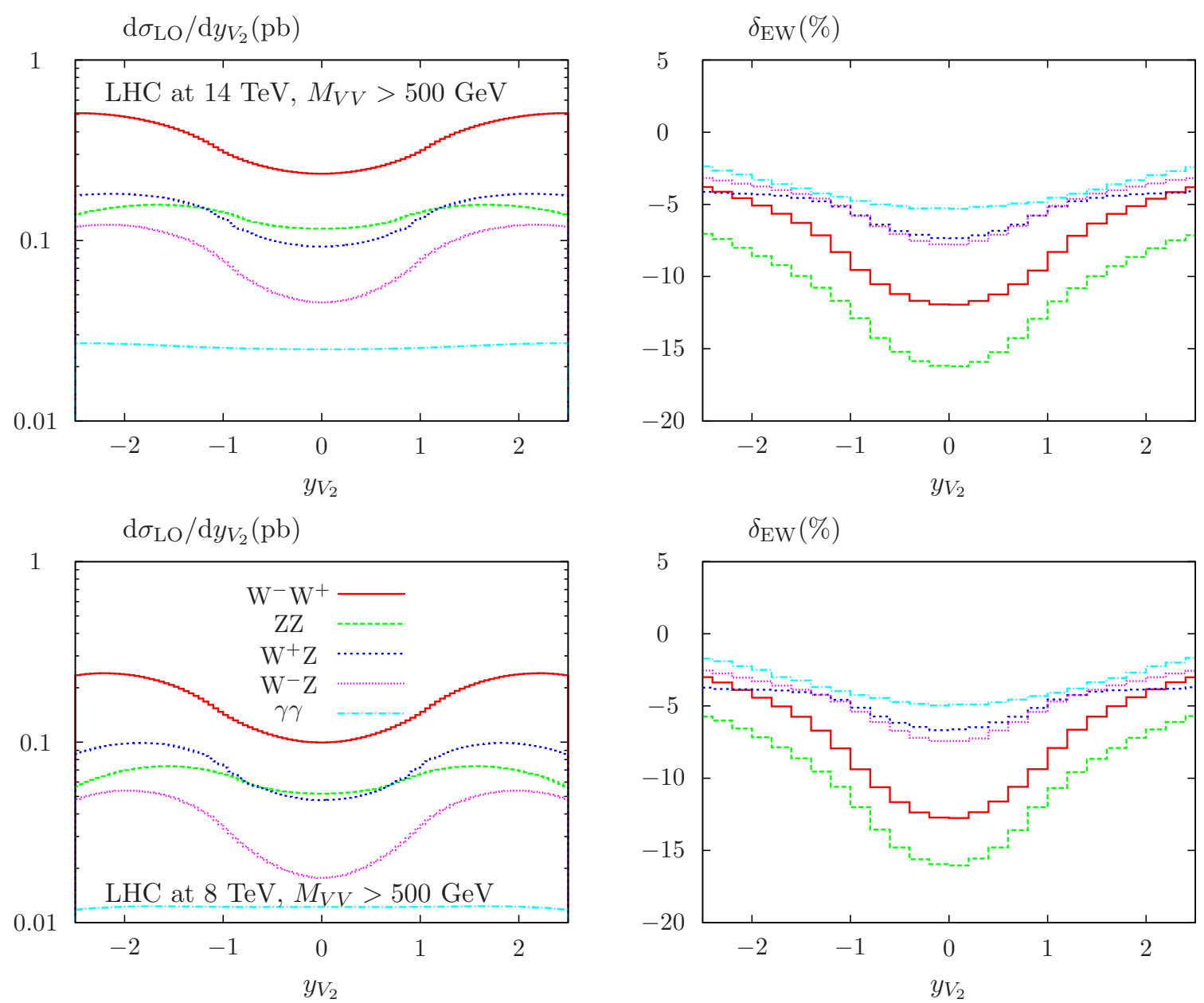

Figure 9. Differential LO distributions of the boson rapidity (left) and corresponding EW corrections (right) at LHC14 (top) and LHC8 (bottom) for a minimal invariant mass of $500 \mathrm{GeV}$.

Let us now consider vector-boson pair production at highest energies accessible at the LHC. This is achieved by restricting $M_{V V}$ to values above $500 \mathrm{GeV}$ (fig. 9) and $1 \mathrm{TeV}$ (fig. 10). Looking at the right panels of figure 9 and figure 10, we observe that the relative EW corrections to the boson rapidity distributions are sizable at low rapidities, corresponding to central events with high transverse momenta, i.e. the Sudakov region. Large rapidities, in contrast, correspond to small scattering angles (small $|\hat{t}|$ or $|\hat{u}|$ ) and thus small $p_{\mathrm{T}}$. EW corrections per se are logarithmically enhanced only in the Sudakov region. For identical cuts on the invariant mass $M_{V V}$ the corrections are very similar at LHC14 and LHC8, respectively, reaching $-15 \%(-30 \%)$ for $M_{V V}>500 \mathrm{GeV}\left(M_{V V}>1000 \mathrm{GeV}\right)$. The corresponding corrections for the distributions with respect to $\Delta y_{V V}=y_{V_{1}}-y_{V_{2}}$ were presented in figures 3 and 4 for $M_{V V}>500 \mathrm{GeV}\left(M_{V V}>1000 \mathrm{GeV}\right)$ for LHC8 (LHC14). Large corrections were observed for small rapidity gaps, amounting to nearly $-40 \%(-25 \%)$ for central ZZ production at LHC14 (LHC8).

This behaviour can be pushed even further by going to $M_{V V}>1.5 \mathrm{TeV}$, a region 

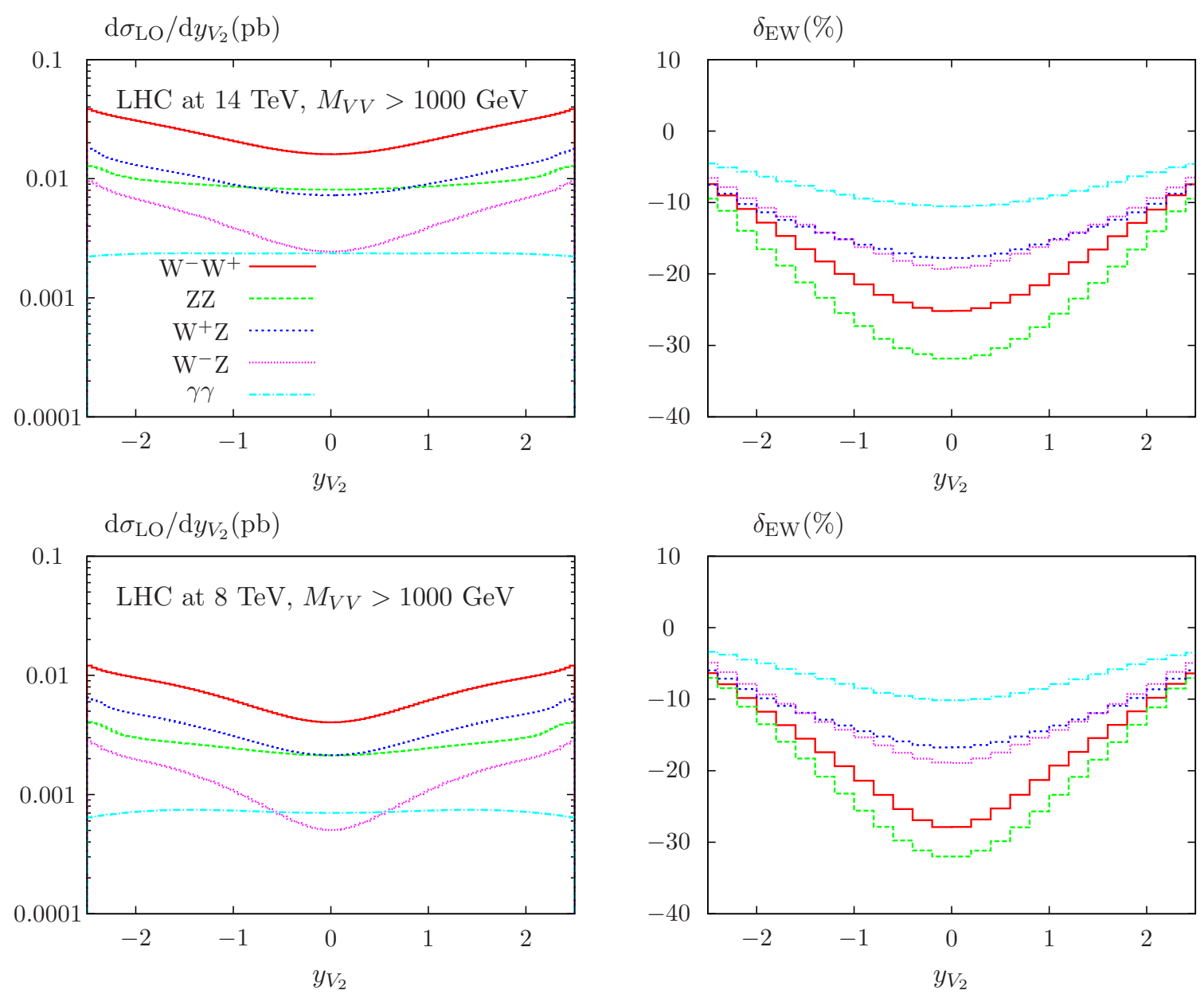

Figure 10. Differential LO distributions of the boson rapidity (left) and corresponding EW corrections (right) at LHC14 (top) and LHC8 (bottom) for a minimal invariant mass of $1000 \mathrm{GeV}$.

potentially accessible at a high luminosity LHC. Results for a minimal invariant mass of $M_{V V}>750 \mathrm{GeV}\left(M_{V V}>1500 \mathrm{GeV}\right)$ at LHC8 (LHC14) are shown in figure 11, and for Z-pair production at LHC14 in this kinematic region the corresponding corrections reach $-50 \%$. In this regime, also weak two-loop effects might be required to reliably predict the cross sections.

\subsubsection{Comparison with existing results}

To additionally validate our numerical analysis and to assess the remaining theoretical uncertainties, we compare our predictions for vector-boson pair production at hadron colliders with older results obtained in a high-energy approximation [4]. Although a tuned comparison in general is not possible since the approach taken in the present work does not allow to apply event-selection cuts to the leptonic decay products of the vector bosons, as is done in ref. [4], (See, however, the discussion in section 3.4.), qualitative statements can be made already now. 

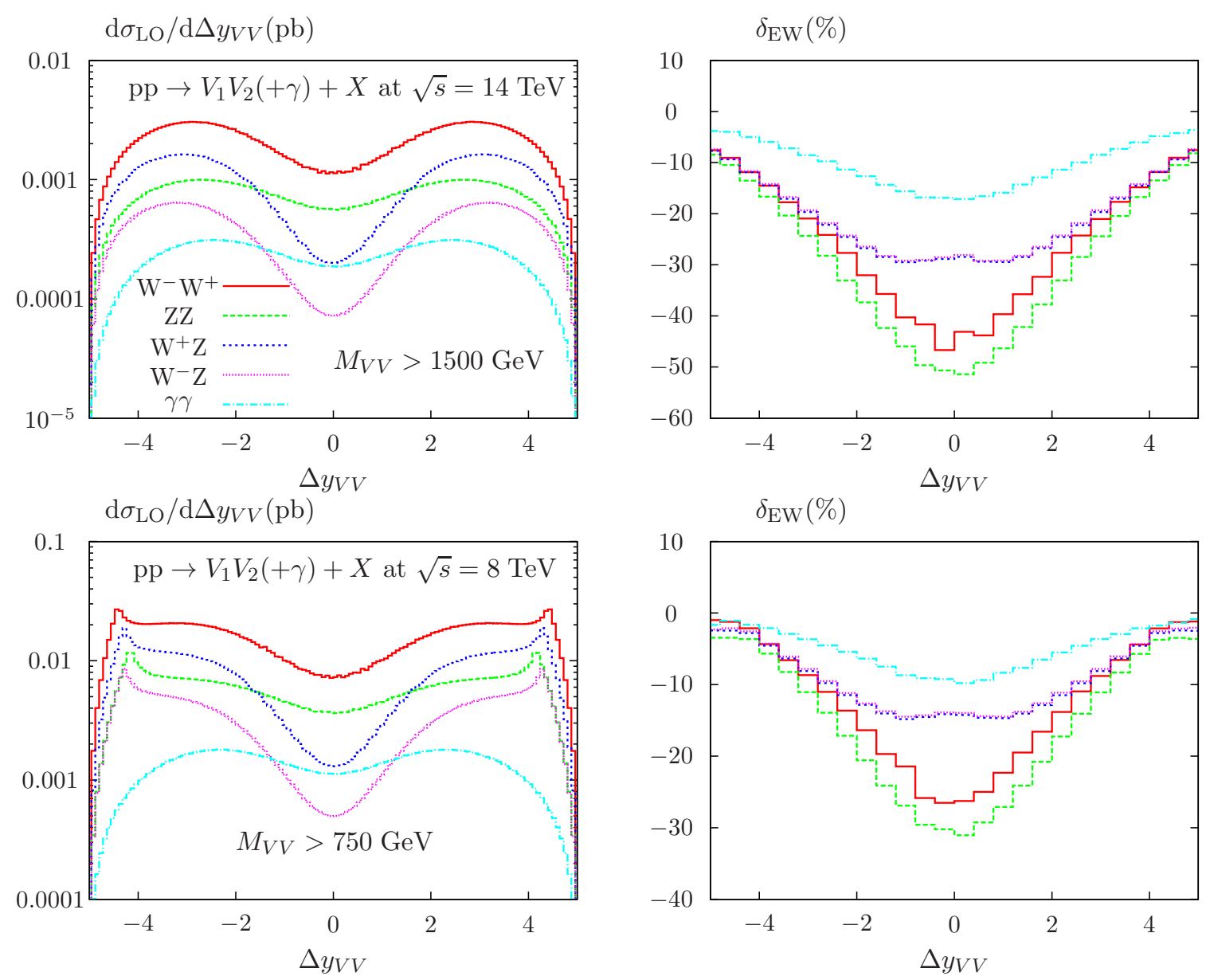

Figure 11. Differential LO distributions of the boson rapidity gap (left) and corresponding EW corrections (right) for a minimal invariant mass of $1500 \mathrm{GeV}$ at LHC14 (top) and $750 \mathrm{GeV}$ at LHC8 (bottom), respectively.

Comparing our results for Z-pair production $\left(\delta_{\mathrm{EW}}^{\mathrm{ZZ}}\right)$ to those given in Table 3 of ref. [4] $\left(\delta_{\mathrm{EW}}^{\mathrm{ZZ}, \mathrm{ADK}}\right)$, we observe very good agreement in the whole energy range considered if we employ the additional constraint $\left|\Delta y_{\mathrm{ZZ}}\right|<3$ on the rapidity gap of the $\mathrm{Z}$ bosons to explicitly enforce Sudakov kinematics (i.e. $\hat{s},|\hat{t}|,|\hat{u}| \gg M_{\mathrm{Z}}^{2}$ ). For instance, we find $\delta_{\mathrm{EW}}^{\mathrm{ZZ}}=-28.5 \%$, to be compared with $\delta_{\mathrm{EW}}^{\mathrm{ZZ}, \mathrm{ADK}}=-28.1 \%$ for $M_{\mathrm{ZZ}}>1000 \mathrm{GeV}$. As expected, EW corrections to $\mathrm{ZZ}$ production in the Sudakov regime are exhaustively described by logarithmic weak corrections, and mass effects do not play a significant role. Surprisingly, also off-shell effects and final-state photon radiation, both included in Ref. [4], as well as LHC acceptance cuts, do not seem to noticeably affect the relative $\mathrm{EW}$ corrections after the $\mathrm{Z}$ reconstruction has been performed.

Turning to WZ production, the comparison is also straightforward. According to scenario (7.3) of ref. [4] we apply-in addition to our default cuts-a cut on the transverse momentum of the $\mathrm{Z}$ boson and obtain $\delta_{\mathrm{EW}}^{\mathrm{W}}+\mathrm{Z}=-22.4 \%$ for $p_{\mathrm{T}, \mathrm{Z}}>500 \mathrm{GeV}$, which is in reasonable agreement with $\delta_{\mathrm{EW}}^{\mathrm{WZ}, \mathrm{ADK}}=-21.2 \%$ given in table 1 of ref. [4]. 


\subsection{Z-pair production: polarization and decays}

Once sufficiently large samples of gauge-boson pairs have been produced, it will be important to investigate the angular distributions of their decay products, which evidently carry the information about the $\mathrm{Z}$ (or $\mathrm{W}$ ) polarization.

Up to the order considered in the present paper, real plus virtual photon radiation can be separated in a gauge-invariant manner, as far as $\mathrm{ZZ}$ production is concerned. It is quite remarkable that this purely electromagnetic subset of the corrections to $\mathrm{ZZ}$ production is tiny, in general below $1 \%$ in all cases discussed in this paper. In a first step we thus evaluate the cross sections for the production of polarized $\mathrm{Z}$ pairs and the impact of purely weak corrections on these cross sections. In a second step, we consider combined production and decay for the mode $\mathrm{pp} \rightarrow \mathrm{Z}\left(\rightarrow \mathrm{e}^{+} \mathrm{e}^{-}\right) \mathrm{Z}\left(\rightarrow \mu^{+} \mu^{-}\right)+X$, including weak corrections.

\subsubsection{Polarization effects}

Let us start with polarized Z-pair production at LHC8 and LHC14. Longitudinal, rightand left-circular production are denoted by $(\mathrm{L}),(+)$ and $(-)$. The cross sections for the production of one $\mathrm{Z}$ with polarization $(i)$ and one with polarization $(j)$ is represented by $\sigma(i j)$. The unpolarized cross section is thus composed of the following combination

$$
\sigma_{\mathrm{tot}}=\sigma(\mathrm{LL})+\sigma(++)+\sigma(--)+\sigma(+-)+\sigma(\mathrm{L}+)+\sigma(\mathrm{L}-) .
$$

As a consequence of CP symmetry $\sigma(\mathrm{L}+)=\sigma(\mathrm{L}-)$ and $\sigma(++)=\sigma(--)$. The remaining four independent combinations are listed in table 7 . The results are presented for the default cuts, for events with $p_{\mathrm{T}, \mathrm{Z}}>500 \mathrm{GeV}$ and for $p_{\mathrm{T}, \mathrm{Z}}>1000 \mathrm{GeV}$.

Let us first discuss the Born cross section, which is the upper entry for each partial or summed cross section. Already for the default cuts it receives its major contribution (70\%) from the $(+-)$ configuration, for larger transverse momenta the remaining configurations die out quickly. This behaviour can be deduced directly from the equivalence theorem: neutral scalar pair production is strictly forbidden, which in the present case leads to the $M_{\mathrm{Z}}^{2} / \hat{s}$ suppression of $\sigma(\mathrm{LL})$. Also the $(++)$ configuration is strictly forbidden for massless gauge bosons. The $M_{\mathrm{Z}} / \sqrt{\hat{s}}$ behaviour of $\sigma(\mathrm{L} \pm)$ is similar to the one for $\mathrm{W}$ pairs e.g. discussed in ref. [24]. Qualitatively, this behaviour can indeed be read off from a comparison of the ratios $\sigma(\mathrm{LL}) / \sigma(+-)$ and $\sigma(\mathrm{L} \pm) / \sigma(+-)$ for the $p_{\mathrm{T}}^{\text {cut }}$ values of $500 \mathrm{GeV}$ and $1000 \mathrm{GeV}$.

Also shown in table 7 as lower entry are the $\mathcal{O}(\alpha)$ weak corrections $\delta \sigma_{\text {weak }}$. Again we observe the overall reduction by about $5 \%$, arising mainly from small $\hat{s}$, which is fairly similar for the different polarizations, as far as low $\hat{s}$ are concerned. For larger $p_{\mathrm{T}}$ values a different pattern emerges. For the suppressed diagonal configurations (LL), $(++)$, $(--)$ the negative corrections increase with $p_{\mathrm{T}}$, and quickly exceed the Born contribution. Hence, if one would try to analyze the different polarizations separately, one should include the squared 1-loop correction. The corresponding values for $\delta \sigma_{\text {weak }}$ representing the interference between Born and one-loop term plus one-loop squared contribution are given in round brackets in the same line. However, since all these contributions are below one permille, they are irrelevant for all practical considerations. Note, in addition, that the 


\begin{tabular}{|c|c|c|c|c|c|}
\hline \multicolumn{6}{|c|}{$\mathrm{pp} \rightarrow \mathrm{ZZ}+X$} \\
\hline ZZ polarizations & summed & $\mathrm{LL}$ & $\mathrm{L}+$ & ++ & +- \\
\hline \multicolumn{6}{|c|}{ LHC14 } \\
\hline $\begin{array}{c}\text { default cuts } \\
\sigma_{\mathrm{LO}} / \mathrm{pb} \\
\delta \sigma_{\text {weak }} / \mathrm{pb}\end{array}$ & $\begin{array}{c}7.067 \\
-0.338(-0.292)\end{array}$ & $\begin{array}{c}0.402 \\
-0.015(-0.014)\end{array}$ & $\begin{array}{c}0.734 \\
-0.029(-0.025)\end{array}$ & $\begin{array}{c}0.100 \\
-0.004(-0.003)\end{array}$ & $\begin{array}{c}4.997 \\
-0.257(-0.223)\end{array}$ \\
\hline $\begin{array}{c}p_{\mathrm{T}, \mathrm{Z}}>500 \mathrm{GeV} \\
\sigma_{\mathrm{LO}} / \mathrm{pb} \\
\delta \sigma_{\text {weak }} / \mathrm{pb}\end{array}$ & $\begin{array}{c}10^{-2} \times[0.499 \\
-0.195(-0.148)]\end{array}$ & $\begin{array}{c}10^{-7} \times[0.921 \\
-4.70(+5.577)]\end{array}$ & $\begin{array}{c}10^{-4} \times[0.334 \\
-0.087(-0.067)]\end{array}$ & $\begin{array}{c}10^{-7} \times[0.230 \\
-0.426(-0.185)]\end{array}$ & $\begin{array}{c}10^{-2} \times[0.492 \\
-0.192(-0.147)]\end{array}$ \\
\hline $\begin{array}{c}p_{\mathrm{T}, \mathrm{Z}}>1000 \mathrm{GeV} \\
\sigma_{\mathrm{LO}} / \mathrm{pb} \\
\delta \sigma_{\text {weak }} / \mathrm{pb}\end{array}$ & $\begin{array}{c}10^{-3} \times[0.146 \\
-0.088(-0.062)]\end{array}$ & $\begin{array}{c}10^{-9} \times[0.189 \\
-4.319(+30.04)]\end{array}$ & $\begin{array}{c}10^{-6} \times[0.306 \\
-0.126(-0.090)]\end{array}$ & $\begin{array}{c}10^{-10} \times[0.475 \\
-2.953(+2.295)]\end{array}$ & $\begin{array}{c}10^{-3} \times[0.146 \\
-0.088(-0.062)]\end{array}$ \\
\hline \multicolumn{6}{|c|}{ LHC8 } \\
\hline $\begin{array}{c}\sigma_{\mathrm{LO}} / \mathrm{pb} \\
\delta \sigma_{\text {weak }} / \mathrm{pb}\end{array}$ & $\begin{array}{c}3.810 \\
-0.179(-0.155) \\
\end{array}$ & $\begin{array}{c}0.223 \\
-0.009(-0.008) \\
\end{array}$ & $\begin{array}{c}0.396 \\
-0.016(-0.014)\end{array}$ & $\begin{array}{c}10^{-1} \times[0.559 \\
-0.002(-0.002)]\end{array}$ & $\begin{array}{c}2.676 \\
-0.134(-0.117) \\
\end{array}$ \\
\hline $\begin{array}{c}p_{\mathrm{T}, \mathrm{Z}}>500 \mathrm{GeV} \\
\sigma_{\mathrm{LO}} / \mathrm{pb} \\
\delta \sigma_{\text {weak }} / \mathrm{pb}\end{array}$ & $\begin{array}{c}10^{-2} \times[0.101 \\
-0.039(-0.030)\end{array}$ & $\begin{array}{c}10^{-7} \times[0.202 \\
-0.975(+0.748)]\end{array}$ & $\begin{array}{c}10^{-5} \times[0.779 \\
-0.204(-0.157)]\end{array}$ & $\begin{array}{c}10^{-8} \times[0.504 \\
-0.895(-0.425)]\end{array}$ & $\begin{array}{c}10^{-3} \times[0.996 \\
-0.383(-0.293) \\
\end{array}$ \\
\hline $\begin{array}{c}p_{\mathrm{T}, \mathrm{Z}}>1000 \mathrm{GeV} \\
\sigma_{\mathrm{LO}} / \mathrm{pb} \\
\delta \sigma_{\text {weak }} / \mathrm{pb}\end{array}$ & $\begin{array}{c}10^{-5} \times[0.919 \\
-0.557(-0.387)\end{array}$ & $\begin{array}{c}10^{-10} \times[0.121 \\
-2.599(+14.909)]\end{array}$ & $\begin{array}{c}10^{-7} \times[0.231 \\
-0.098(-0.070)]\end{array}$ & $\begin{array}{c}10^{-11} \times[0.303 \\
-1.742(+1.043)]\end{array}$ & $\begin{array}{r}10^{-5} \times[0.915 \\
-0.555(-0.387)\end{array}$ \\
\hline
\end{tabular}

Table 7. Polarized LO cross sections and corresponding weak corrections to ZZ production at the LHC for different cuts on the boson transverse momenta. The first entry for the corrections represents the interference between Born and one-loop amplitude, the second entry (in brackets) includes the squared one-loop amplitude.

residual uncertainties on the integrated cross sections due to missing higher-order weak corrections are at the level of $10 \%$ and $20 \%$ for $p_{\mathrm{T}, \mathrm{Z}}>500 \mathrm{GeV}$ and $p_{\mathrm{T}, \mathrm{Z}}>1000 \mathrm{GeV}$, respectively.

In total a fairly simple pattern emerges: for small $p_{\mathrm{T}}$ electroweak corrections are very similar for all polarizations and can be taken as one global factor, for large $p_{\mathrm{T}}$ only one combination survives and corrections are again trivially represented by one factor. Electroweak corrections can therefore be represented for the bulk of events at each $\hat{s}$ and $\hat{t}$ by a correction factor which does not modify the relative importance of the different polarizations.

\subsubsection{Leptonic decays}

Let us, in a next step, evaluate the complete production and decay process for the $\mathrm{e}^{+} \mathrm{e}^{-} \mu^{+} \mu^{-}$ final state at the LHC. For the acceptance cuts on muon and electron transverse momenta and rapidities we adopt the prescriptions of ref. [4], namely

$$
p_{\mathrm{T}, l}>15 \mathrm{GeV}, \quad\left|y_{l}\right|<3 .
$$

The intermediate $\mathrm{Z}$ bosons are reconstructed from the final-state leptons requiring

$$
\left|M_{l \bar{l}}-M_{\mathrm{Z}}\right|<20 \mathrm{GeV}
$$


to suppress the admixture of virtual photons and to improve the validity of the approximations used to compute the weak corrections.

The cuts employed in table 8 are chosen to mimic on the one hand the experimental acceptance, and on the other hand, select events with increasing $\sqrt{\hat{s}}$, corresponding to the invariant mass $M_{\mathrm{inv}}(4 l)$ of the four-lepton system. The first four columns represent predictions for LHC14 and LHC8 using four variants of the Born approximation. In the first column we give the full LO cross section, including all off-shell effects and non-resonant contributions, in a naive fixed-width implementation using non-vanishing constant vectorboson widths ${ }^{5}$

$$
\Gamma_{\mathrm{Z}}=2.505044 \mathrm{GeV}, \quad \Gamma_{\mathrm{W}}=2.099360 \mathrm{GeV}
$$

in propagators with time-like 4-momenta,

$$
\frac{1}{p^{2}-M_{V}^{2}} \rightarrow \frac{1}{p^{2}-M_{V}^{2}+\theta\left(p^{2}\right) \mathrm{i} M_{V} \Gamma_{V}} .
$$

The second column shows the corresponding results evaluated in the complex-mass scheme (CMS) [25] which involves complex couplings. In addition, we present results for the Born cross section in the double-pole approximation (DPA) as described in ref. [4] and in the narrow-width approximation (NWA), where resonant propagators are replaced according to

$$
\frac{1}{\left(p^{2}-M_{V}^{2}\right)^{2}+M_{V}^{2} \Gamma_{V}^{2}} \rightarrow \frac{\pi}{M_{V} \Gamma_{V}} \delta\left(p^{2}-M_{V}^{2}\right)
$$

corresponding to the limit $\Gamma_{V} / M_{V} \rightarrow 0$. Both approximations only include doubly-resonant contributions. Note that both the $\gamma^{*}$ and Z-induced amplitudes are included in the full LO predictions, while the $\gamma^{*}$-mediated diagrams are absent in the approximate results. The relative weak corrections, which are identical to a level of $1 \%$ between DPA and NWA, are listed in column 5 .

With the cuts employed until now a sizable fraction of events corresponds to large $\hat{s}$ but small $|\hat{t}|$, and the Sudakov approximation is not applicable. Imposing, however, a cut on the rapidity difference between the reconstructed $\mathrm{Z}$ bosons, $\left|\Delta y_{\mathrm{ZZ}}\right|<3$, removes events with small scattering angle and decreases the total rates by roughly $30 \%$ at high $M_{\text {inv }}(4 l)$. Moreover, it leads to enhanced EW corrections, as shown in table 9. We point out that the results of table 9 are in good agreement (better than $2 \%$ ) with those presented in table 3 of ref. [4], despite the fact that we do not include QED corrections and corresponding non-factorizable contributions which arise in the DPA.

\subsection{Real-radiation contributions}

Let us finally investigate the phenomenological effect of additional massive-boson radiation as defined in section 2.3. In figures 12 and 13 we show the corresponding relative corrections for LHC14 and LHC8, respectively. As far as WW, ZZ and $\mathrm{W}^{+} \mathrm{Z}$ production at LHC14 is concerned, the contributions, which are always below $10 \%$, are found to be of minor importance for the phenomenological analysis, and the effects are even smaller

\footnotetext{
${ }^{5}$ For the numerical evaluation we adopt the values from ref. [4] for $\Gamma_{\mathrm{Z}}$ and $\Gamma_{\mathrm{W}}$.
} 


\begin{tabular}{|c|c|c|c|c|c|}
\hline \multicolumn{6}{|c|}{$\mathrm{pp} \rightarrow\left(\mathrm{Z} / \gamma^{*}\right)\left(\mathrm{Z} / \gamma^{*}\right)+X \rightarrow \mathrm{e}^{+} \mathrm{e}^{-} \mu^{+} \mu^{-}+X$} \\
\hline$M_{\text {inv }}^{\text {cut }}(4 l) / \mathrm{GeV}$ & $\sigma_{\mathrm{LO}}^{\text {naive }} / \mathrm{pb}$ & $\sigma_{\mathrm{LO}}^{\mathrm{CMS}} / \mathrm{pb}$ & $\sigma_{\mathrm{LO}}^{\mathrm{DPA}} / \mathrm{pb}$ & $\sigma_{\mathrm{LO}}^{\mathrm{NWA}} / \mathrm{pb}$ & $\delta_{\text {weak }}^{\text {DPA }} / \%$ \\
\hline \multicolumn{6}{|c|}{ LHC14 } \\
\hline 200 & $0.835 \times 10^{-2}$ & $0.835 \times 10^{-2}$ & $0.815 \times 10^{-2}$ & $0.875 \times 10^{-2}$ & -5.4 \\
\hline 300 & $0.239 \times 10^{-2}$ & $0.239 \times 10^{-2}$ & $0.233 \times 10^{-2}$ & $0.249 \times 10^{-2}$ & -8.1 \\
\hline 400 & $0.987 \times 10^{-3}$ & $0.987 \times 10^{-3}$ & $0.966 \times 10^{-3}$ & $1.035 \times 10^{-3}$ & -10.2 \\
\hline 500 & $0.484 \times 10^{-3}$ & $0.484 \times 10^{-3}$ & $0.473 \times 10^{-3}$ & $0.508 \times 10^{-3}$ & -12.4 \\
\hline 600 & $0.261 \times 10^{-3}$ & $0.261 \times 10^{-3}$ & $0.256 \times 10^{-3}$ & $0.275 \times 10^{-3}$ & -14.5 \\
\hline 700 & $0.151 \times 10^{-3}$ & $0.151 \times 10^{-3}$ & $0.148 \times 10^{-3}$ & $0.159 \times 10^{-3}$ & -16.6 \\
\hline 800 & $0.920 \times 10^{-4}$ & $0.920 \times 10^{-4}$ & $0.901 \times 10^{-4}$ & $0.971 \times 10^{-4}$ & -18.8 \\
\hline 900 & $0.584 \times 10^{-4}$ & $0.584 \times 10^{-4}$ & $0.572 \times 10^{-4}$ & $0.617 \times 10^{-4}$ & -20.8 \\
\hline 1000 & $0.384 \times 10^{-4}$ & $0.384 \times 10^{-4}$ & $0.376 \times 10^{-4}$ & $0.406 \times 10^{-4}$ & -22.9 \\
\hline \multicolumn{6}{|c|}{ LHC8 } \\
\hline 200 & $\mid 0.445 \times 10^{-2}$ & $0.445 \times 10^{-2}$ & $0.435 \times 10^{-2}$ & $0.466 \times 10^{-2}$ & -5.3 \\
\hline 300 & $0.120 \times 10^{-3}$ & $0.120 \times 10^{-3}$ & $0.117 \times 10^{-2}$ & $0.125 \times 10^{-3}$ & -7.7 \\
\hline 400 & $0.463 \times 10^{-3}$ & $0.463 \times 10^{-3}$ & $0.452 \times 10^{-3}$ & $0.484 \times 10^{-3}$ & -9.4 \\
\hline 500 & $0.210 \times 10^{-3}$ & $0.210 \times 10^{-3}$ & $0.206 \times 10^{-3}$ & $0.221 \times 10^{-3}$ & -11.2 \\
\hline 600 & $0.105 \times 10^{-3}$ & $0.105 \times 10^{-3}$ & $0.103 \times 10^{-3}$ & $0.110 \times 10^{-3}$ & -12.9 \\
\hline 700 & $0.557 \times 10^{-3}$ & $0.557 \times 10^{-3}$ & $0.544 \times 10^{-3}$ & $0.586 \times 10^{-3}$ & -14.8 \\
\hline 800 & $0.309 \times 10^{-4}$ & $0.309 \times 10^{-4}$ & $0.302 \times 10^{-4}$ & $0.326 \times 10^{-4}$ & -16.6 \\
\hline 900 & $0.178 \times 10^{-4}$ & $0.178 \times 10^{-4}$ & $0.174 \times 10^{-4}$ & $0.186 \times 10^{-4}$ & -18.5 \\
\hline 1000 & $0.106 \times 10^{-4}$ & $0.106 \times 10^{-4}$ & $0.103 \times 10^{-4}$ & $0.111 \times 10^{-4}$ & -20.4 \\
\hline
\end{tabular}

Table 8. LO cross section for Z-boson pair production with 4-lepton final states and corresponding weak corrections at the LHC for different cut values of the 4-lepton invariant mass.

at $\mathrm{LHC} 8$, as expected. In contrast to this, in the case of $\mathrm{W}^{-} \mathrm{Z}$ production we observe remarkably large corrections reaching $+25 \%(+15 \%)$ at LHC14 (LHC8). However, we point out that this effect, though sizable, cannot be attributed to large logarithms arising from the infrared structure of the corresponding squared matrix elements. In fact, it can be easily understood recalling that the corresponding real-radiation contributions as defined in section 2.3 include $\mathrm{W}^{-} \mathrm{ZW}^{+}$production at $\mathrm{LO}$ which, compared to $\mathrm{W}^{-} \mathrm{Z}$ production is from the beginning enhanced by a factor of 2 due to one u-quark PDF factor. To verify this line of argumentation, we have checked that for the Tevatron the relative corrections for $\mathrm{W}^{ \pm} \mathrm{Z}$ production indeed coincide, not exceeding the level of $+5 \%$ for $p_{\mathrm{T}}^{\text {cut }}=300 \mathrm{GeV}$. Nevertheless, the numerical effects discussed here can easily (and definitely should be) taken into account in the experimental analysis of the background.

Turning to the effects of hard photon radiation displayed in the lower two plots of figures 12 and 13, respectively, we find that the corresponding contributions are moderate and by far largest for $\mathrm{W}$-pair production, exceeding $+5 \%$, while they are completely irrelevant for Z-pair production. 


\begin{tabular}{|c|c|c|c|c|c|}
\hline \multicolumn{6}{|c|}{$\mathrm{pp} \rightarrow\left(\mathrm{Z} / \gamma^{*}\right)\left(\mathrm{Z} / \gamma^{*}\right)+X \rightarrow \mathrm{e}^{+} \mathrm{e}^{-} \mu^{+} \mu^{-}+X,|\Delta y \mathrm{ZZ}|<3$} \\
\hline$M_{\text {inv }}^{\text {cut }}(4 l) / \mathrm{GeV}$ & $\sigma_{\mathrm{LO}}^{\text {naive }} / \mathrm{pb}$ & $\sigma_{\mathrm{LO}}^{\mathrm{CMS}} / \mathrm{pb}$ & $\sigma_{\mathrm{LO}}^{\text {DPA }} / \mathrm{pb}$ & $\sigma_{\mathrm{LO}}^{\text {NWA }} / \mathrm{pb}$ & $\delta_{\text {weak }}^{\text {DPA }} / \%$ \\
\hline \hline \multicolumn{6}{|l|}{} \\
\hline \multicolumn{6}{|c|}{$\mathrm{LHC} 14$} \\
\hline 200 & $0.815 \times 10^{-2}$ & $0.815 \times 10^{-2}$ & $0.795 \times 10^{-2}$ & $0.855 \times 10^{-2}$ & -5.4 \\
300 & $0.219 \times 10^{-2}$ & $0.219 \times 10^{-2}$ & $0.214 \times 10^{-2}$ & $0.229 \times 10^{-2}$ & -8.4 \\
400 & $0.791 \times 10^{-3}$ & $0.791 \times 10^{-3}$ & $0.770 \times 10^{-3}$ & $0.828 \times 10^{-3}$ & -11.6 \\
500 & $0.326 \times 10^{-3}$ & $0.326 \times 10^{-3}$ & $0.319 \times 10^{-3}$ & $0.343 \times 10^{-3}$ & -15.9 \\
600 & $0.168 \times 10^{-3}$ & $0.168 \times 10^{-3}$ & $0.164 \times 10^{-3}$ & $0.177 \times 10^{-3}$ & -19.3 \\
700 & $0.962 \times 10^{-4}$ & $0.962 \times 10^{-4}$ & $0.941 \times 10^{-4}$ & $1.017 \times 10^{-4}$ & -22.3 \\
800 & $0.587 \times 10^{-4}$ & $0.587 \times 10^{-4}$ & $0.575 \times 10^{-4}$ & $0.621 \times 10^{-4}$ & -24.9 \\
900 & $0.374 \times 10^{-4}$ & $0.374 \times 10^{-4}$ & $0.367 \times 10^{-4}$ & $0.397 \times 10^{-4}$ & -27.4 \\
1000 & $0.247 \times 10^{-4}$ & $0.247 \times 10^{-4}$ & $0.242 \times 10^{-4}$ & $0.262 \times 10^{-4}$ & -29.7 \\
\hline
\end{tabular}

Table 9. LO cross section for Z-boson pair production with 4-lepton final states and corresponding weak corrections at LHC14 for different cut values of the 4-lepton invariant mass in the Sudakov regime with $\left|\Delta y_{\mathrm{zz}}\right|<3$.

\section{Conclusions}

We have computed the full one-loop electroweak corrections to on-shell $\mathrm{ZZ}, \mathrm{W}^{ \pm} \mathrm{Z}, \mathrm{ZZ}$ and $\gamma \gamma$ production at hadron colliders, for the first time consistently taking into account all mass effects. Furthermore, the results presented are not limited to a particular kinematic regime, allowing for flexible predictions valid in all regions of phase space. In case of ZZ production we have also included the leptonic decays and the corresponding weak corrections, finding good agreement with former computations restricted to Sudakov kinematics. The relative corrections are negative and grow with increasing center-of-mass energy. They are largest for $\mathrm{ZZ}$ production, reaching $-50 \%$ at energies accessible at LHC14, and smallest for $\gamma \gamma$ production. As an interesting new finding we observe that the relative corrections are not only sizable in the Sudakov regime as has been shown before, but may also - in the ZZ case - give significant contributions at rather low transverse momenta. We have also investigated the effect of massive boson radiation processes which may be considered as background to vector-boson pair production depending on the details of the experimental setup. The effects are smaller than expected from naive partonic considerations and may easily be included in the experimental studies. Our predictions rely on the experimental reconstruction of the intermediate bosons. In the future leptonic decays of the vector bosons will be included also in the analysis of WW and WZ production together with the corresponding $\mathcal{O}(\alpha)$ corrections to allow for a more realistic event definition.

\section{Acknowledgements}

This work has been supported by "Strukturiertes Promotionskolleg Elementarteilchen- und Astroteilchenphysik", SFB TR9 "Computational and Particle Physics" and BMBF Contract 05HT4VKATI3. 

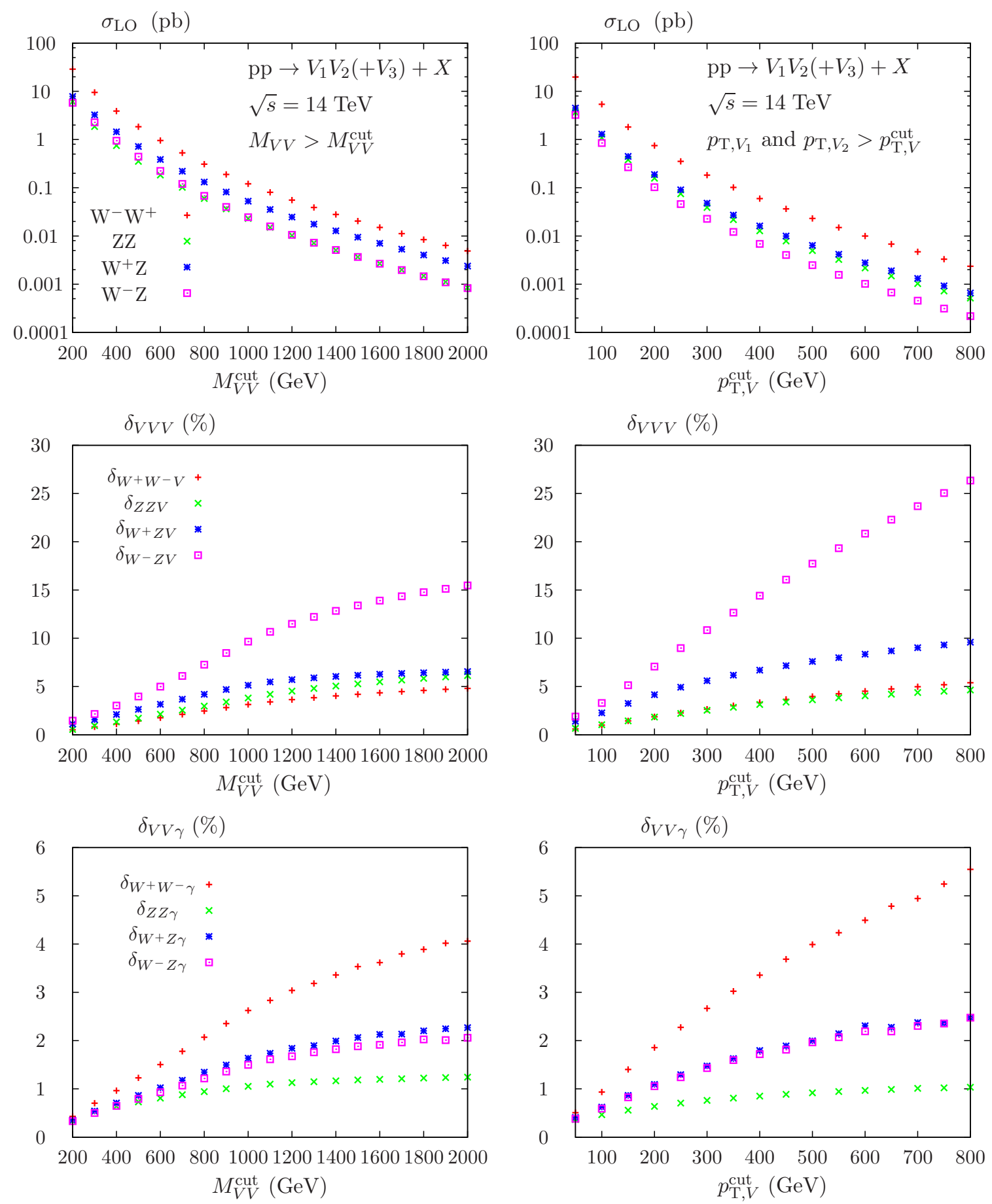

Figure 12. Integrated LO cross sections (top) and relative corrections at the LHC14 due to radiation of one additional massive vector boson (center) and hard-photon radiation (bottom) evaluated with our default setup for different cuts on the invariant mass (left)/transverse momenta (right) of the final-state bosons. 

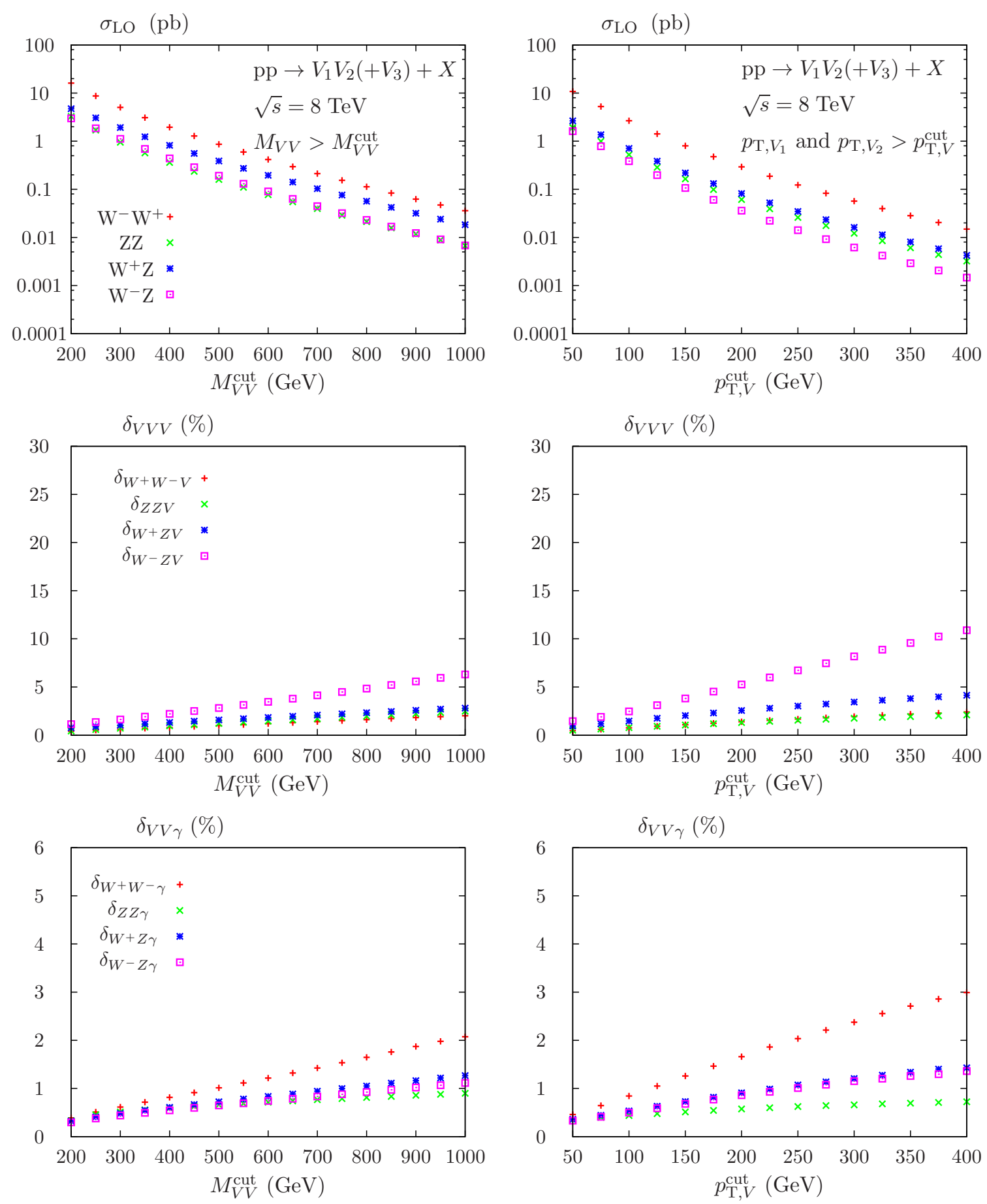

Figure 13. Integrated LO cross sections (top) and relative corrections at the LHC8 due to radiation of one additional massive vector boson (center) and hard-photon radiation (bottom) evaluated with our default setup for different cuts on the invariant mass (left)/transverse momenta (right) of the final-state bosons. 


\section{References}

[1] A. Bierweiler, T. Kasprzik, H. Kühn and S. Uccirati, JHEP 1211 (2012) 093 [arXiv:1208.3147 [hep-ph]].

[2] A. Bierweiler, T. Kasprzik and J. H. Kühn, PoS(ICHEP2012)078 [arXiv:1208.3404 [hep-ph]].

[3] E. Accomando, A. Denner and S. Pozzorini, Phys. Rev. D 65 (2002) 073003 [hep-ph/0110114].

[4] E. Accomando, A. Denner and A. Kaiser, Nucl. Phys. B 706 (2005) 325 [hep-ph/0409247].

[5] E. Accomando, A. Denner, C. Meier, Eur. Phys. J. C47 (2006) 125-146. [hep-ph/0509234].

[6] J. H. Kühn, F. Metzler, A. A. Penin and S. Uccirati, JHEP 1106 (2011) 143 [arXiv:1101.2563 [hep-ph]].

[7] J. Küblbeck, M. Böhm and A. Denner, Comput. Phys. Commun. 60 (1990) 165; H. Eck and J. Küblbeck, Guide to FeynArts 1.0, University of Würzburg, 1992.

[8] T. Hahn, Comput. Phys. Commun. 140 (2001) 418 [hep-ph/0012260].

[9] T. Hahn and M. Pérez-Victoria, Comput. Phys. Commun. 118 (1999) 153 [hep-ph/9807565].

[10] T. Hahn, C. Schappacher, Comput. Phys. Commun. 143 (2002) 54-68. [hep-ph/0105349].

[11] G. J. van Oldenborgh, J. A. M. Vermaseren, Z. Phys. C46 (1990) 425-438.

[12] Nogueira, P. Automatic Feynman graph generation. J. Comput. Phys., Vol. 105 (1993) pp. 279-289.

[13] J. A. M. Vermaseren, math-ph/0010025.

[14] S. Dittmaier, M. Krämer, Phys. Rev. D65 (2002) 073007. [hep-ph/0109062].

[15] K. P. O. Diener, S. Dittmaier and W. Hollik, Phys. Rev. D 69 (2004) 073005 [hep-ph/0310364].

[16] A. Denner, Fortsch. Phys. 41 (1993) 307-420. [arXiv:0709.1075 [hep-ph]].

[17] W. J. Stirling and E. Vryonidou, arXiv:1212.6537 [hep-ph].

[18] A. D. Martin et al., Eur. Phys. J. C63, (2009) 189-285. [arXiv:0901.0002 [hep-ph]].

[19] M. R. Whalley, D. Bourilkov and R. C. Group, in HERA and the LHC, eds. A. de Roeck and H. Jung (CERN-2005-014, Geneva, 2005), p. 575, hep-ph/0508110.

[20] A. D. Martin, R. G. Roberts, W. J. Stirling, R. S. Thorne, Eur. Phys. J. C39 (2005) 155-161. [hep-ph/0411040].

[21] M. Roth and S. Weinzierl, Phys. Lett. B 590 (2004) 190 [hep-ph/0403200].

[22] J. Layssac and F. M. Renard, Phys. Rev. D 64 (2001) 053018 [hep-ph/0104205].

[23] A. Denner, S. Dittmaier and M. Strobel, Phys. Rev. D 53 (1996) 44 [hep-ph/9507372].

[24] W. Beenacker, A. Denner and F. A. Berends, In *Munich/Annecy/Hamburg 1991, Proceedings, e+ e- collisions at 500-GeV, pt. A* 151-164

[25] A. Denner, S. Dittmaier, M. Roth and L. H. Wieders, Nucl. Phys. B 724 (2005) 247 [hep-ph/0505042]; A. Denner and S. Dittmaier, Nucl. Phys. Proc. Suppl. 160 (2006) 22 [hep-ph/0605312]. 